\title{
Aspects of interface elasticity theory
}

\author{
Ali Javili $^{\mathrm{a}}$, Niels Saabye Ottosen ${ }^{\mathrm{b}}$, Matti Ristinmaa ${ }^{\mathrm{b}}$, Jörn Mosler ${ }^{\mathrm{c}, *}$ \\ ${ }^{a}$ Department of Mechanical Engineering, Bilkent University, 06800 Ankara, Turkey \\ ${ }^{b}$ Division of Solid Mechanics, Lund University, SE-22100 Lund, Sweden \\ ${ }^{c}$ Institute of Mechanics, TU Dortmund, D-44227 Dortmund, Germany
}

\begin{abstract}
Interfaces significantly influence the overall material response especially when the area-to-volume ratio is large, for instance in nanocrystalline solids. A well-established and frequently applied framework suitable for modeling interfaces dates back to the pioneering work by Gurtin and Murdoch (1975) on surface elasticity theory and its generalization to interface elasticity theory. In this contribution, interface elasticity theory is revisited and different aspects of this theory are carefully examined. Two alternative formulations based on stress vectors and stress tensors are given to unify various existing approaches in this context. Focus is on the hyper-elastic mechanical behavior of such interfaces. Interface elasticity theory at finite deformation is critically reanalyzed and several subtle conclusions are highlighted. Finally, a consistent linearized interface elasticity theory is established. We propose an energetically consistent interface linear elasticity theory together with its appropriate stress measures.
\end{abstract}

Keywords: Interface elasticity, Linearized elasticity, Coherent interfaces, Surface shear, Gurtin-Murdoch theory

\section{Introduction}

Almost all materials, at some scale of observation, are made from different constituents. The transition

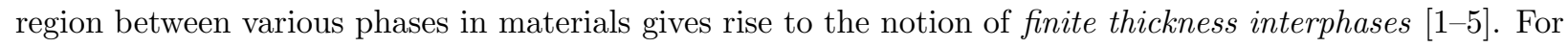
practical purposes, an interphase can be sufficiently approximated by a zero-thickness interface model when its thickness is relatively small compared to other length scales, thereby the interface is a two-dimensional manifold representing the finite thickness interphase. In order to capture the interphase behavior, the interface is endowed with its own energy density per unit area following the original ideas of Gibbs. This approach leads to a particular in-plane elasticity theory on the interface pioneered by Gurtin and Murdoch in their seminal work on surface elasticity theory [6]. Shortly afterwards, Murdoch [7] established an interface

\footnotetext{
* Corresponding author

Email addresses: ajavili@bilkent.edu.tr (Ali Javili), Niels_Saabye.Ottosen@solid.Ith.se (Niels Saabye Ottosen), Matti.Ristinmaa@solid.lth.se (Matti Ristinmaa), joern.mosler@tu-dortmund.de (Jörn Mosler)
} 
elasticity theory as a general form of the surface elasticity theory [6]. The interface elasticity theory treats the interface essentially as a two-sided surface and thus, it is nearly identical to the surface elasticity theory.

The area-to-volume ratio is proportional to the inverse of the dimension for geometrically equivalent objects. With decreasing scale follows an increasing area-to-volume ratio where interface elasticity theory plays an increasingly important role on the overall behavior of materials resulting in size effects. With the emerging applications of nanomaterials 8,13 , and the utility of surface elasticity theory to capture the behavior of solids at the nano-scale and particularly the size effect [14 23] the importance of interface elasticity theory has dramatically increased. As an example, whereas the yearly number of citations to the work of Gurtin and Murdoch [6] during the period 1975-2000 amounted to a few, the yearly number of citations grew exponentially after year 2000 and the total number of citations up to 2015 exceeds 1000 . Figure 1 illustrates the citation record of [6].

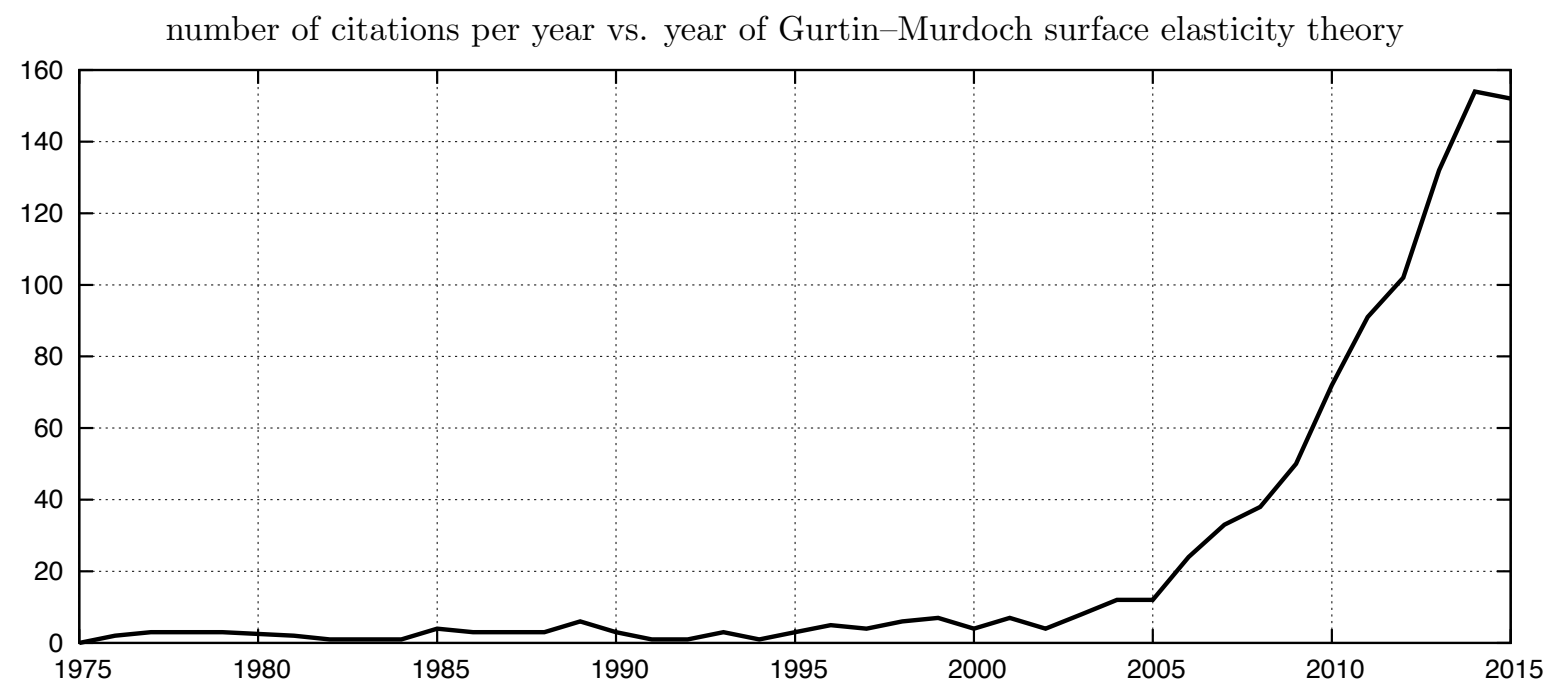

Figure 1: Citation record of the seminal work of Gurtin and Murdoch 6] on surface elasticity theory published in 1975. The total number of citations up to 2015 exceeds 1000. The exponential increase of the citations is probably associated with emerging applications of nano-materials. The data to produce this graph was obtained from Scopus.

The principal assumption of interface elasticity theory is to allow the zero-thickness coherent interface to have its own thermodynamic structures per area; this applies to the Helmholtz energy, dissipation and the like. This assumption results in an interface stress along the interface and consequently a traction jump across the interface while the displacements remain continuous. Such interfaces are referred to as thermodynamic singular surfaces by Daher and Maugin [24. The governing equations of such interfaces simplify to the generalized Young-Laplace equation [25] 27], see [28 39] and references therein for further details.

While interface elasticity theory is widely applied to explain the material response at the nano-scale, certain aspects of the theory are still not well understood and require further clarifications. The implications 
of interface elasticity theory are essentially due to the two-dimensional nature of the problem in a threedimensional embedding space. Curvilinear coordinates and fundamental concepts of differential geometry are vital to appropriately formulate interface elasticity [40 42]. Without a proper tensorial notation based on convected coordinates, detailed contributions have recently emerged to explain relatively simple geometrical concepts, see for instance [43. In this manuscript, we employ (covariant) tensorial notation and elaborate on the consequences of this more convenient framework. Within a finite deformation setting, several elusive conclusions associated with interface elasticity theory are highlighted. Furthermore, we establish an interface elasticity theory for small strains via linearizing the finite strain version and hence, guarantee the consistency between the two versions. We show that the controversial non-symmetric gradient term in the linear interface stress appears naturally through linearization of the geometrically exact interface elasticity theory.

In passing, we mention that interface elasticity theory may be understood as the exact opposite to the cohesive interface model introduced by Barenblatt [44, 45, Dugdale [46] and Hillerborg [47. In contrast to the elastic interface model, the cohesive interface model allows for displacement jumps but the traction remains continuous across the interface. Cohesive interface models, have been extensively studied in [48,62] from both theoretical and computational aspects with various applications and traction-separation laws.

\section{Key contributions of this manuscript}

The objective of this manuscript is twofold. First, we establish a finite deformation interface elasticity theory. In doing so, we formulate the problem in two alternative formats based on stress vectors and stress tensors to unify diverse notations in the literature. Several exquisite consequences below, implied by interface elasticity theory, are discussed:

- Superficiality of the interface deformation gradient implies tangentiality but the same analogy does not hold for the interface Piola stress.

- Interface Piola stress is superficial by definition but not necessarily tangential.

- Interface Piola stress is tangential due to angular momentum balance.

- Dependence of the energy on the interface normal leads to the notion of surface shear.

- Material frame indifference as well as balance of angular momentum rule out the dependence of the interface Helmholtz energy on the interface normal.

- Material frame indifference implies balance of angular momentum.

- Isotropic interface Helmholtz energy is expressed in terms of the two invariants of the interface right Cauchy-Green tensor. 
Secondly, we establish a consistent linear interface elasticity theory. The most important outcome is to show that the controversial non-symmetric gradient term in the interface stress is meaningful and derives from a consistent linearization of the geometrically non-linear interface elasticity theory. Furthermore, the non-symmetric part is a consequence of the fact that the stress-free configuration does not coincide with the strain-free configuration.

\section{Organization of this manuscript}

This manuscript is organized as follows. After briefly introducing the notations and definitions, Section 2 deals with the kinematics of coherent material interfaces within a finite deformation setting. The geometrically exact interface elasticity theory is briefly formulated in Section 3 whereby the main ingredients are balance equations and constitutive laws. The geometrically exact interface elasticity theory is linearized in Section 4. The linearized interface elasticity theory is particularly relevant to applications in nano-materials and atomistic simulations. Section 5 concludes this work and discusses possible further research work.

\section{Notations and definitions}

The contents of this manuscript are heavily based on the differential geometry of interfaces as two-dimensional manifolds within the three-dimensional space. Preliminaries of the differential geometry of interfaces are briefly reviewed in Appendix A. Here, $\{\bar{\bullet}\}$ refers to an interface variable with its bulk counterpart being $\{\bullet\}$. Following this convention throughout the manuscript, surface, interface and curve quantities are denoted as $\{\widehat{\bullet}\},\{\boldsymbol{\bullet}\}$ and $\{\widetilde{\bullet}\}$, respectively and are therefore distinguishable from the bulk quantity $\{\bullet\}$ by an accent on top. Direct notation is adopted throughout. Occasional use is made of index notation, the summation convention for repeated indices being implied. The jump of the quantity $\{\bullet\}$ across the interface is defined by $\llbracket\{\bullet\} \rrbracket=\{\bullet\}^{+}-\{\bullet\}^{-}$.

\section{Kinematics of interfaces}

Consider a continuum body that takes the material configuration $\mathcal{B}_{0}$ at time $t=0$ and the spatial configuration $\mathcal{B}_{t}$ at any time $t$ as shown in the Fig. 2. The interface $\mathcal{I}_{0}$ splits the material configuration $\mathcal{B}_{0}$ into two disjoint subdomains $\mathcal{B}_{0}^{-}$and $\mathcal{B}_{0}^{+}$. Analogously, interface $\mathcal{I}_{t}$ in the spatial configuration is the common boundary of the two subdomains $\mathcal{B}_{t}^{-}$and $\mathcal{B}_{t}^{+}$. The outward unit normal to the boundary of the material configuration $\mathcal{B}_{0}$ is denoted $\widehat{\boldsymbol{N}}$. The unit normal to the interface pointing from the minus to the plus side is denoted $\overline{\boldsymbol{N}}$ in the material configuration. The outward unit normal to the boundary of the interface $\partial \mathcal{I}_{0}$ but tangential to the interface $\mathcal{I}_{0}$ is denoted $\widetilde{\boldsymbol{N}}$. In the spatial configuration, the surface, interface and curve normals are denoted $\widehat{\boldsymbol{n}}, \overline{\boldsymbol{n}}$ and $\widetilde{\boldsymbol{n}}$, respectively. It proves convenient to define the interface identity tensor $\overline{\boldsymbol{I}}:=\boldsymbol{I}-\overline{\boldsymbol{N}} \otimes \overline{\boldsymbol{N}}$ in the material configuration as the projector onto the interface. In contrast to the bulk 
identity tensor $\boldsymbol{i}=\boldsymbol{I}$, the interface identity in the spatial configuration $\overline{\boldsymbol{i}}:=\boldsymbol{i}-\overline{\boldsymbol{n}} \otimes \overline{\boldsymbol{n}}$ does not necessarily coincides with its material counterpart.

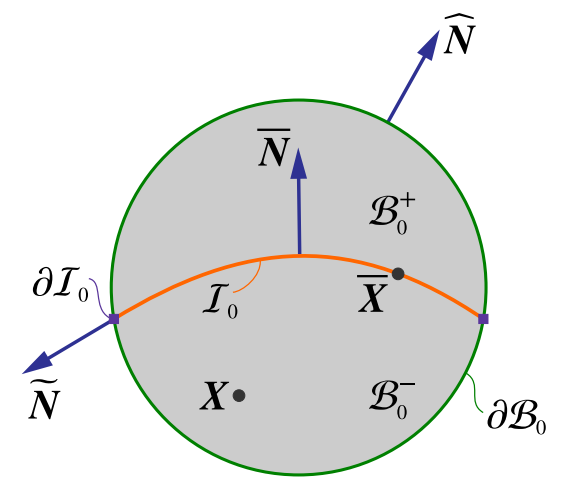

material configuration

time $t=0$
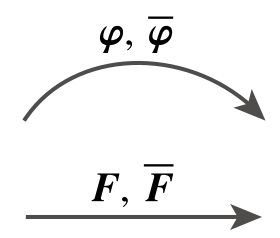

$\partial \mathcal{B}_{t}$

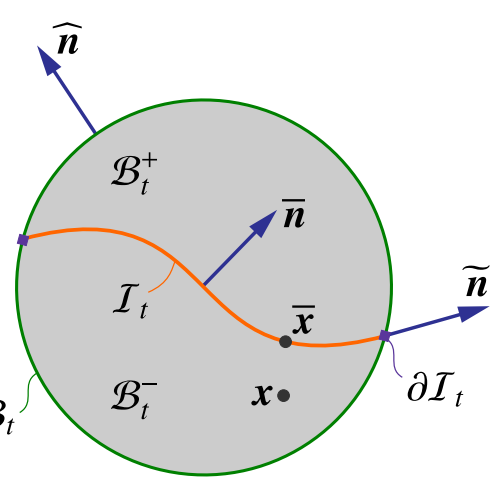

spatial configuration

time $t>0$

Figure 2: The material and spatial configuration of a continuum body and its associated motion and deformation gradient. The two sides of the body in the material configuration, $\mathcal{B}_{0}^{-}$and $\mathcal{B}_{0}^{+}$, are bonded via interface $\mathcal{I}_{0}$. The placements of the particles in the material configuration $\boldsymbol{X}$ are mapped to their spatial counterpart $\boldsymbol{x}$ via the non-linear deformation map $\boldsymbol{x}=\boldsymbol{\varphi}(\boldsymbol{X})$. The interface is material in the sense that $\overline{\boldsymbol{\varphi}}=\left.\boldsymbol{\varphi}\right|_{\mathcal{I}_{0}}$ and it is also coherent, that is $\llbracket \boldsymbol{x} \rrbracket=\mathbf{0}$.

The placement of material particles in the bulk and on the interface are labeled $\boldsymbol{X}$ and $\overline{\boldsymbol{X}}$, respectively, in the material configuration. The motions from the material to the spatial configuration in the bulk and on the interface are denoted as $\varphi$ and $\bar{\varphi}$, respectively. In the spatial configuration, the placement of material particles in the bulk and on the interface are labeled $\boldsymbol{x}$ and $\overline{\boldsymbol{x}}$, respectively. The placements of particles in the spatial configuration are related to their counterparts in the material configuration via the non-linear deformation maps $\varphi$ and $\bar{\varphi}$ as

$$
\boldsymbol{x}=\boldsymbol{\varphi}(\boldsymbol{X}) \quad \forall X \in \mathcal{B}_{0} \quad, \quad \overline{\boldsymbol{x}}=\bar{\varphi}(\bar{X}) \quad \forall \bar{X} \in \mathcal{I}_{0} .
$$

Henceforth, we assume the interface to be material such that it follows the motion of the bulk or more precisely $\bar{\varphi}=\left.\varphi\right|_{\mathcal{I}_{0}}$. Furthermore, the interface is assumed to be coherent in the sense that the motion jump across the interface vanishes or that $\llbracket \varphi \rrbracket=0$.

The deformation gradient in the bulk, denoted $\boldsymbol{F}$, is a linear deformation map that relates an infinitesimal line element $\mathrm{d} \boldsymbol{X} \in T \mathcal{B}_{0}$ to its spatial counterpart $\mathrm{d} \boldsymbol{x} \in T \mathcal{B}_{t}$ via the relation $\mathrm{d} \boldsymbol{x}=\boldsymbol{F} \cdot \mathrm{d} \boldsymbol{X}$ whereby $\boldsymbol{F}=\operatorname{Grad} \boldsymbol{\varphi}$. Similarly to the bulk, we define the interface deformation gradient $\overline{\boldsymbol{F}}$ as the linear map between the infinitesimal line element $\mathrm{d} \overline{\boldsymbol{X}} \in T \mathcal{I}_{0}$ and $\mathrm{d} \overline{\boldsymbol{x}} \in T \mathcal{I}_{t}$ with $\mathrm{d} \overline{\boldsymbol{x}}=\overline{\boldsymbol{F}} \cdot \mathrm{d} \overline{\boldsymbol{X}}$ whereby $\overline{\boldsymbol{F}}=\overline{\operatorname{Grad}} \overline{\boldsymbol{\varphi}}$. Note, $\overline{\operatorname{Grad}}\{\bullet\}$ denotes the interface gradient operator defined by $\overline{\operatorname{Grad}}\{\bullet\}:=\operatorname{Grad}\{\bullet\} \cdot \overline{\boldsymbol{I}}$ as the projection 
of the gradient operator onto the interface. It then follows that

$$
\boldsymbol{F}=\boldsymbol{g}_{i} \otimes \boldsymbol{G}^{i} \quad \text { with } \quad i \in\{1,2,3\} \quad, \quad \overline{\boldsymbol{F}}=\overline{\boldsymbol{g}}_{\alpha} \otimes \overline{\boldsymbol{G}}^{\alpha} \quad \text { with } \quad \alpha \in\{1,2\},
$$

in which $\boldsymbol{g}_{i}$ and $\overline{\boldsymbol{g}}_{\alpha}$ are the covariant base vectors in the spatial configuration, and where $\boldsymbol{G}^{i}$ and $\overline{\boldsymbol{G}}^{\alpha}$ are the conravariant base vectors in the material configuration.

Let $\mathrm{d} V$ and $\mathrm{d} v$ denote the volume elements of the bulk in the material and spatial configurations, respectively. Similarly, $\mathrm{d} A$ and $\mathrm{d} a$ denote the area elements of the interface in the material and spatial configurations, respectively. The ratios of volume elements and area elements in the spatial over the material configuration are denoted $J$ and $\bar{J}$, respectively, as

$$
J=\mathrm{d} v / \mathrm{d} V \quad \text { with } \quad J:=\operatorname{Det} \boldsymbol{F} \quad, \quad \bar{J}=\mathrm{d} a / \mathrm{d} A \quad \text { with } \quad \bar{J}:=\overline{\operatorname{Det}} \overline{\boldsymbol{F}} .
$$

From the view point of classic continuum mechanics, the area element on the interface in the material configuration $\mathrm{d} \boldsymbol{A}=\mathrm{d} A \overline{\boldsymbol{N}}$ maps to its spatial counterpart $\mathrm{d} \boldsymbol{a}=\mathrm{d} a \overline{\boldsymbol{n}}$ according to the Nanson's formula $\mathrm{d} \boldsymbol{a}=J \boldsymbol{F}^{-\mathrm{t}} \cdot \mathrm{d} \boldsymbol{A}$. The line element $\mathrm{d} \boldsymbol{L}$ tangential to the interface and normal to the boundary of the interface in the material configuration maps to its spatial counterpart via the interface normal map $\overline{\operatorname{Cof}} \overline{\boldsymbol{F}}^{=} \bar{J} \overline{\boldsymbol{F}}^{\text {-t }}$ as $\mathrm{d} \boldsymbol{l}=\overline{\operatorname{Cof}} \overline{\boldsymbol{F}} \cdot \mathrm{d} \boldsymbol{L}$ in which $\mathrm{d} \boldsymbol{L}=\mathrm{d} L \widetilde{\boldsymbol{N}}$ and $\mathrm{d} \boldsymbol{l}=\mathrm{d} l \widetilde{\boldsymbol{n}}$.

\section{Geometrically exact interface elasticity theory}

The objective of this section is to briefly formulate the interface elasticity theory within a finite deformation setting. In particular, balance equations and constitutive laws of interface elasticity theory are established in what follows. Detailed expositions on non-linear continuum mechanics can be found in 63 65] among others. Further details on formulation of interfaces can be found in the references listed in the introduction.

\subsection{Balance equations}

The balance equations are derived by viewing configuration $\mathcal{B}_{0}$ as the entire continuum body or as an arbitrary cutout volume of the continuum body. This view is only assumed to reduce the notations and it does not alter the derivations nor the final equations. To proceed, we first write the global external mechanical power $\mathcal{P}_{\mathrm{ex}}^{\mathrm{gl}}$ in an integral form

$$
\begin{aligned}
\mathcal{P}_{\mathrm{ex}}^{\mathrm{gl}}=\int_{\mathcal{B}_{0}^{-}} \dot{\boldsymbol{\varphi}} \cdot \boldsymbol{b}_{0} \mathrm{~d} V+\int_{\mathcal{B}_{0}^{+}} \dot{\boldsymbol{\varphi}} \cdot \boldsymbol{b}_{0} \mathrm{~d} V+\int_{\partial \mathcal{B}_{0}^{-}} \dot{\boldsymbol{\varphi}} \cdot \widehat{\boldsymbol{b}}_{0} \mathrm{~d} A \\
+\int_{\partial \mathcal{B}_{0}^{+}} \dot{\boldsymbol{\varphi}} \cdot \widehat{\boldsymbol{b}}_{0} \mathrm{~d} A+\int_{\partial \mathcal{I}_{0}} \dot{\bar{\varphi}} \cdot \widetilde{\boldsymbol{b}}_{0} \mathrm{~d} L,
\end{aligned}
$$

in which $\dot{\varphi}$ and $\dot{\bar{\varphi}}$ denote the material time derivatives of the bulk and interface motion $\varphi$ and $\bar{\varphi}$, respectively. Note, that boundaries $\partial \mathcal{B}_{0}^{-}$and $\partial \mathcal{B}_{0}^{+}$follow the same motion as the bulk itself in the sense of kinematic slavery and, thus, the surface is material. The same analogy holds for interface $\mathcal{I}_{0}$ as well as for the boundary 
of interface $\partial \mathcal{I}_{0}$. The force density of the bulk per unit volume in the material configuration is denoted $\boldsymbol{b}_{0}$. Similarly, the force density of the surface per unit area in the material configuration is denoted $\widehat{\boldsymbol{b}}_{0}$, often referred to as traction vector. In a similar way, the force density of the curve per unit length in the material configuration is denoted $\widetilde{\boldsymbol{b}}_{0}$, often referred to as a traction-like vector. The interface is assumed to be completely flexible to bending, and thus, no bending moments or twisting moments exist in the interface.

Following Cauchy theorem type arguments, surface traction $\widehat{\boldsymbol{b}}_{0}$ can be related to the Piola stress $\boldsymbol{P}$ in the bulk through surface normal $\widehat{\boldsymbol{N}}$ according to $\widehat{\boldsymbol{b}}_{0}=\boldsymbol{P} \cdot \widehat{\boldsymbol{N}}$. Interface elasticity theory is based on Cauchy theorem type arguments for a two-dimensional manifold. Bearing this in mind, the interface is provided with its own Piola stress $\overline{\boldsymbol{P}}$, and traction $\widetilde{\boldsymbol{b}}_{0}$ on the boundary of the interface $\partial \mathcal{I}_{0}$ is related to the interface stress via $\widetilde{\boldsymbol{b}}_{0}=\overline{\boldsymbol{P}} \cdot \widetilde{\boldsymbol{N}}$. The interface stress $\overline{\boldsymbol{P}}$ is superficial in the sense that it possesses the property $\overline{\boldsymbol{P}} \cdot \overline{\boldsymbol{N}}=\mathbf{0}$. The superficiality of the interface stress is a crucial property in this context. It can be shown that the superficiality property is the consequence of a first-order continuum theory [66, 67] and the zero-thickness interface [68. Rewriting Eq. (4) in terms of stresses instead of tractions yields

$$
\begin{aligned}
\mathcal{P}_{\mathrm{ex}}^{\mathrm{gl}}=\mathcal{P}_{\mathrm{ex}}^{\mathrm{gl}}(\dot{\boldsymbol{\varphi}}, \dot{\bar{\varphi}})= & \int_{\mathcal{B}_{0}^{-}} \dot{\boldsymbol{\varphi}} \cdot \boldsymbol{b}_{0} \mathrm{~d} V+\int_{\mathcal{B}_{0}^{+}} \dot{\boldsymbol{\varphi}} \cdot \boldsymbol{b}_{0} \mathrm{~d} V \\
& +\int_{\partial \mathcal{B}_{0}^{-}} \dot{\boldsymbol{\varphi}} \cdot \boldsymbol{P} \cdot \widehat{\boldsymbol{N}} \mathrm{d} A+\int_{\partial \mathcal{B}_{0}^{+}} \dot{\boldsymbol{\varphi}} \cdot \boldsymbol{P} \cdot \widehat{\boldsymbol{N}} \mathrm{d} A+\int_{\partial \mathcal{I}_{0}} \dot{\bar{\varphi}} \cdot \overline{\boldsymbol{P}} \cdot \widetilde{\boldsymbol{N}} \mathrm{d} L .
\end{aligned}
$$

Secondly, we impose the invariance of the global external mechanical power $\mathcal{P}_{\text {ex }}^{\mathrm{gl}}$ with respect to superposed rigid body motions as

$$
\mathcal{P}_{\mathrm{ex}}^{\mathrm{gl}}=\mathcal{P}_{\mathrm{ex}}^{\mathrm{gl}}(\dot{\boldsymbol{\varphi}}, \dot{\bar{\varphi}}) \stackrel{!}{=} \mathcal{P}_{\mathrm{ex}}^{\mathrm{gl}}(\dot{\boldsymbol{\varphi}}+\boldsymbol{v}+\boldsymbol{\omega} \times \boldsymbol{x}, \dot{\bar{\varphi}}+\boldsymbol{v}+\boldsymbol{\omega} \times \overline{\boldsymbol{x}}) \quad \forall \boldsymbol{v}, \boldsymbol{\omega}
$$

where the constant, but otherwise arbitrary linear and angular velocities are denoted $\boldsymbol{v}$ and $\boldsymbol{\omega}$, respectively. To be more precise, neglecting inertia and body forces 11 the invariance with respect to translations renders the global form of balance of linear momentum

$$
\int_{\partial \mathcal{B}_{0}^{-}} \boldsymbol{v} \cdot \boldsymbol{P} \cdot \widehat{\boldsymbol{N}} \mathrm{d} A+\int_{\partial \mathcal{B}_{0}^{+}} \boldsymbol{v} \cdot \boldsymbol{P} \cdot \widehat{\boldsymbol{N}} \mathrm{d} A+\int_{\partial \mathcal{I}_{0}} \boldsymbol{v} \cdot \overline{\boldsymbol{P}} \cdot \widetilde{\boldsymbol{N}} \mathrm{d} L=0 \quad \forall \boldsymbol{v},
$$

and the invariance with respect to rotations renders the global form of balance of angular momentum

$$
\int_{\partial \mathcal{B}_{0}^{-}}[\boldsymbol{\omega} \times \boldsymbol{x}] \cdot \boldsymbol{P} \cdot \widehat{\boldsymbol{N}} \mathrm{d} A+\int_{\partial \mathcal{B}_{0}^{+}}[\boldsymbol{\omega} \times \boldsymbol{x}] \cdot \boldsymbol{P} \cdot \widehat{\boldsymbol{N}} \mathrm{d} A+\int_{\partial \mathcal{I}_{0}}[\boldsymbol{\omega} \times \overline{\boldsymbol{x}}] \cdot \overline{\boldsymbol{P}} \cdot \widetilde{\boldsymbol{N}} \mathrm{d} L=0 \quad \forall \boldsymbol{\omega} .
$$

Thirdly, through localization of the global balances (7) and (8) to an infinitesimal subdomain in the bulk, the classic balance of linear and angular momentum in the bulk are obtained as

$$
\operatorname{Div} \boldsymbol{P}=\mathbf{0} \quad \text { and } \quad \varepsilon:\left[\boldsymbol{F} \cdot \boldsymbol{P}^{\mathrm{t}}\right]=\mathbf{0} \Leftrightarrow \boldsymbol{P} \cdot \boldsymbol{F}^{\mathrm{t}}=\boldsymbol{F} \cdot \boldsymbol{P}^{\mathrm{t}},
$$

\footnotetext{
${ }^{1}$ For simplicity, quasi-static problems are considered.
} 
respectively, with $\varepsilon$ being the third-order permutation tensor. Along the same lines, via localization to an infinitesimal subdomain on the interface, the balance of linear and of angular momentum on the interface are obtained as

$$
\overline{\operatorname{Div}} \overline{\boldsymbol{P}}+\llbracket \boldsymbol{P} \rrbracket \cdot \overline{\boldsymbol{N}}=\mathbf{0} \quad \text { and } \quad \varepsilon:\left[\overline{\boldsymbol{F}} \cdot \overline{\boldsymbol{P}}^{\mathrm{t}}\right]=\mathbf{0} \Leftrightarrow \overline{\boldsymbol{P}} \cdot \overline{\boldsymbol{F}}^{\mathrm{t}}=\overline{\boldsymbol{F}} \cdot \overline{\boldsymbol{P}}^{\mathrm{t}},
$$

respectively, in which the interface divergence operator in the material configuration is denoted as $\overline{\operatorname{Div}}\{\bullet\}=$ $\overline{\operatorname{Grad}}\{\bullet\}: \overline{\boldsymbol{I}}$. Note, that the interface divergence operator embeds the information regarding the curvature of the interface, and we therefore do not need to introduce the Christoffel symbol explicitly.

Using the expressions above for balance of the linear momentum, after some mathematical steps, the external power densities in the bulk $\mathcal{P}_{\text {ex }}$ and on the interface $\overline{\mathcal{P}}_{\text {ex }}$ can be written as

$$
\mathcal{P}_{\text {ex }}=\boldsymbol{P}: \dot{\boldsymbol{F}} \quad, \quad \overline{\mathcal{P}}_{\text {ex }}=\overline{\boldsymbol{P}}: \dot{\overline{\boldsymbol{F}}},
$$

where $\{\bullet\}$ denotes the material time derivative of the quantity $\{\bullet\}$. The rates of the deformation gradients in the bulk and on the interface using convected curvilinear coordinates read

$$
\dot{\boldsymbol{F}}=\dot{\boldsymbol{g}}_{i} \otimes \boldsymbol{G}^{i} \quad, \quad \dot{\overline{\boldsymbol{F}}}=\dot{\overline{\boldsymbol{g}}}_{\alpha} \otimes \overline{\boldsymbol{G}}^{\alpha}
$$

In view of Eq. (12), we emphasize that the rate of the interface deformation gradient is superficial but not tangential in the sense that $\dot{\overline{\boldsymbol{F}}} \cdot \overline{\boldsymbol{N}}=\mathbf{0}$ but $\overline{\boldsymbol{n}} \cdot \dot{\overline{\boldsymbol{F}}} \neq \mathbf{0}$. This should be compared with the properties of the interface deformation gradient which is both superficial and tangential, that is $\overline{\boldsymbol{F}} \cdot \overline{\boldsymbol{N}}=\overline{\boldsymbol{n}} \cdot \overline{\boldsymbol{F}}=\mathbf{0}$. This rather peculiar behavior occurs, since the basis vectors $\boldsymbol{g}_{\alpha}$ must lie on the interface but their variation can contain an out of plane component due to the fact that the interface is a two-dimensional manifold within a three-dimensional Euclidean space. By inserting Eq. 12 into the the external power densities [11, and after some manipulations, the external power densities can alternatively be expressed as

$$
\begin{array}{lllll}
\mathcal{P}_{\text {ex }}=\boldsymbol{p}^{i} \cdot \dot{\boldsymbol{g}}_{i} & \text { where } & \boldsymbol{p}^{i}=P^{j i} \boldsymbol{g}_{j} & \text { with } & i, j \in\{1,2,3\}, \\
\overline{\mathcal{P}}_{\text {ex }}=\overline{\boldsymbol{p}}^{\alpha} \cdot \dot{\overline{\boldsymbol{g}}}_{\alpha} & \text { where } & \overline{\boldsymbol{p}}^{\alpha}=\bar{P}^{i \alpha} \overline{\boldsymbol{g}}_{i} & \text { with } & \alpha \in\{1,2\},
\end{array}
$$

in which $\boldsymbol{p}^{i}$ and $\overline{\boldsymbol{p}}^{\alpha}$ are energetically conjugate to the deformation vectors $\boldsymbol{g}_{i}$ and $\overline{\boldsymbol{g}}_{\alpha}$, respectively. Note that $\boldsymbol{P}$ and $\overline{\boldsymbol{P}}$ are stress tensors energetically conjugates to the deformation gradient tensors $\boldsymbol{F}$ and $\overline{\boldsymbol{F}}$, respectively. In an identical way, the quantities $\boldsymbol{p}^{i}$ and $\overline{\boldsymbol{p}}^{\alpha}$ are termed stress vectors henceforth. Piola stress tensors $\boldsymbol{P}$ and $\overline{\boldsymbol{P}}$ are two-point tensors while stress vectors $\boldsymbol{p}^{i}$ and $\overline{\boldsymbol{p}}^{\alpha}$ lie solely on the spatial configuration.

In passing, we mention that, as a dual of Eq. 12 , the rate of the basis vectors $\dot{\boldsymbol{g}}_{i}$ and $\dot{\bar{g}}_{\alpha}$ can be related to the deformation gradients in the bulk and on the interface as

$$
\dot{\boldsymbol{g}}_{i}=\dot{\boldsymbol{F}} \cdot \boldsymbol{G}_{i} \quad, \quad \dot{\overline{\boldsymbol{g}}}_{\alpha}=\dot{\overline{\boldsymbol{F}}} \cdot \overline{\boldsymbol{G}}_{\alpha} \quad \Leftrightarrow \quad \dot{\boldsymbol{F}}=\dot{\boldsymbol{g}}_{i} \otimes \boldsymbol{G}^{i} \quad, \quad \dot{\overline{\boldsymbol{F}}}=\dot{\boldsymbol{g}}_{\alpha} \otimes \overline{\boldsymbol{G}}^{\alpha} .
$$

Furthermore, as a dual of the definitions of the stress vectors based on Piola stress tensors (13), the Piola stress tensors can be related to the stress vectors as

$$
\boldsymbol{P}=\boldsymbol{p}^{i} \otimes \boldsymbol{G}_{i} \quad, \quad \overline{\boldsymbol{P}}=\overline{\boldsymbol{p}}^{\alpha} \otimes \overline{\boldsymbol{G}}_{\alpha} \quad \Leftrightarrow \quad \boldsymbol{p}^{i}=P^{j i} \boldsymbol{g}_{j} \quad, \quad \overline{\boldsymbol{p}}^{\alpha}=\bar{P}^{i \alpha} \overline{\boldsymbol{g}}_{i} .
$$




\subsection{Constitutive laws}

In order to derive the constitutive laws, we start from the external power densities (11) or alternatively (13). We insert the external power densities into the Clausius-Duhem dissipation inequalities

$$
\mathcal{D}=\mathcal{P}_{\text {ex }}-\dot{\psi} \geq 0 \quad, \quad \overline{\mathcal{D}}=\overline{\mathcal{P}}_{\text {ex }}-\dot{\bar{\psi}} \geq 0
$$

in which $\mathcal{D}$ and $\overline{\mathcal{D}}$ denote the dissipation densities in the bulk and on the interface, respectively, in the material configuration. In Clausius-Duhem dissipation inequalities $(16), \psi$ and $\bar{\psi}$ denote the Helmholtz energy densities in the bulk and on the interface, respectively. Replacing the external power in the dissipation inequalities renders

$$
\mathcal{D}=\boldsymbol{P}: \dot{\boldsymbol{F}}-\dot{\psi} \geq 0 \quad, \quad \overline{\mathcal{D}}=\overline{\boldsymbol{P}}: \dot{\overline{\boldsymbol{F}}}-\dot{\bar{\psi}} \geq 0
$$

or alternatively

$$
\mathcal{D}=\boldsymbol{p}^{i} \cdot \dot{\boldsymbol{g}}_{i}-\dot{\psi} \geq 0 \quad, \quad \overline{\mathcal{D}}=\overline{\boldsymbol{p}}^{\alpha} \cdot \dot{\overline{\boldsymbol{g}}}_{\alpha}-\dot{\bar{\psi}} \geq 0 .
$$

The first format for the dissipation inequalities 17 suggests the Helmholtz energies in the bulk and on the interface to be a function of $\boldsymbol{F}$ and $\overline{\boldsymbol{F}}$, respectively. That is, $\psi=\psi(\boldsymbol{F})$ and $\bar{\psi}=\bar{\psi}(\overline{\boldsymbol{F}})$ and thus,

$$
\mathcal{D}=\boldsymbol{P}: \dot{\boldsymbol{F}}-\frac{\partial \psi}{\partial \boldsymbol{F}}: \dot{\boldsymbol{F}}=\left[\boldsymbol{P}-\frac{\partial \psi}{\partial \boldsymbol{F}}\right]: \dot{\boldsymbol{F}} \geq 0 \quad, \quad \overline{\mathcal{D}}=\overline{\boldsymbol{P}}: \dot{\overline{\boldsymbol{F}}}-\frac{\partial \bar{\psi}}{\partial \overline{\boldsymbol{F}}}: \dot{\overline{\boldsymbol{F}}}=\left[\overline{\boldsymbol{P}}-\frac{\partial \psi}{\partial \overline{\boldsymbol{F}}}\right]: \dot{\overline{\boldsymbol{F}}} \geq 0,
$$

and since these equations have to be fulfilled for any time derivative of the deformation gradients, the Piola stresses for fully elastic processes, i.e. $\mathcal{D}=\overline{\mathcal{D}}=0$, are obtained as

$$
\boldsymbol{P}=\frac{\partial \psi}{\partial \boldsymbol{F}} \quad, \quad \overline{\boldsymbol{P}}=\frac{\partial \bar{\psi}}{\partial \overline{\boldsymbol{F}}} .
$$

The second format for the dissipation inequalities 180 suggests the Helmholtz energies in the bulk and on the interface to be a function of $\boldsymbol{g}_{i}$ and $\overline{\boldsymbol{g}}_{\alpha}$, respectively. That is, $\psi=\psi\left(\boldsymbol{g}_{i}\right)$ and $\bar{\psi}=\bar{\psi}\left(\overline{\boldsymbol{g}}_{\alpha}\right)$ and thus,

$$
\mathcal{D}=\boldsymbol{p}^{i} \cdot \dot{\boldsymbol{g}}_{i}-\dot{\psi}=\left[\boldsymbol{p}^{i}-\frac{\partial \psi}{\partial \boldsymbol{g}_{i}}\right] \cdot \dot{\boldsymbol{g}}_{i} \geq 0 \quad, \quad \overline{\mathcal{D}}=\overline{\boldsymbol{p}}^{\alpha} \cdot \dot{\overline{\boldsymbol{g}}}_{\alpha}-\dot{\bar{\psi}}=\left[\overline{\boldsymbol{p}}^{\alpha}-\frac{\partial \bar{\psi}}{\partial \overline{\boldsymbol{g}}_{\alpha}}\right] \cdot \dot{\overline{\boldsymbol{g}}}_{\alpha} \geq 0,
$$

and since these equations must hold for any time derivative of the basis vectors, the stress vectors for fully elastic processes read

$$
\boldsymbol{p}^{i}=\frac{\partial \psi}{\partial \boldsymbol{g}_{i}} \quad, \quad \overline{\boldsymbol{p}}^{\alpha}=\frac{\partial \bar{\psi}}{\partial \overline{\boldsymbol{g}}_{\alpha}} .
$$

\subsection{Material frame indifference}

So far, the balance equations and constitutive laws of interfaces have been established. Here, we study the implications of material frame indifference on the Helmholtz energies $\bar{\psi}(\overline{\boldsymbol{F}})$ or alternatively $\bar{\psi}\left(\overline{\boldsymbol{g}}_{\alpha}\right)$. For the sake of brevity, we limit the discussion exclusively to the interfaces and omit the bulk. 
Let $\boldsymbol{Q} \in \mathcal{S O}(3)$ denote an arbitrary proper orthogonal tensor with the properties $\boldsymbol{Q}^{\mathrm{t}}=\boldsymbol{Q}^{-1}$ and $\operatorname{Det} \boldsymbol{Q}=$ 1. If the interface Helmholtz energy density is expressed in terms of the interface deformation gradient as $\bar{\psi}=\bar{\psi}(\overline{\boldsymbol{F}})$, material frame indifference requires $\bar{\psi}(\overline{\boldsymbol{F}})=\bar{\psi}(\boldsymbol{Q} \cdot \overline{\boldsymbol{F}})$. Therefore, this energy is frame indifferent if and only if the interface deformation gradient $\overline{\boldsymbol{F}}$ enters the energy through the interface right Cauchy-Green tensor $\bar{C}$ as

$$
\bar{\psi}=\bar{\psi}(\overline{\boldsymbol{F}}) \quad \stackrel{\text { material frame indifference }}{=}=\stackrel{=}{=} \quad \bar{\psi}=\bar{\psi}(\overline{\boldsymbol{C}}) \quad \text { with } \quad \overline{\boldsymbol{C}}=\overline{\boldsymbol{F}}^{\mathrm{t}} \cdot \overline{\boldsymbol{F}}=\bar{g}_{\alpha \beta} \overline{\boldsymbol{G}}^{\alpha} \otimes \overline{\boldsymbol{G}}^{\beta} .
$$

Alternatively, the interface Helmholtz energy density can be expressed in terms of the interface covariant bases in the spatial configuration as $\bar{\psi}=\bar{\psi}\left(\overline{\boldsymbol{g}}_{\alpha}\right)$. Material frame indifference requires $\bar{\psi}\left(\overline{\boldsymbol{g}}_{\alpha}\right)=\bar{\psi}\left(\boldsymbol{Q} \cdot \overline{\boldsymbol{g}}_{\alpha}\right)$. Therefore, this energy is frame indifferent if and only if the spatial vectors $\overline{\boldsymbol{g}}_{\alpha}$ enter the energy through the scalar-valued invariants $\bar{g}_{\alpha \beta}$ as

$$
\bar{\psi}=\bar{\psi}\left(\overline{\boldsymbol{g}}_{\alpha}\right) \quad \stackrel{\text { material frame indifference }}{=}=\underline{=}=\bar{\psi}=\bar{\psi}=\bar{\psi}\left(\bar{g}_{\alpha \beta}\right) \quad \text { with } \quad \bar{g}_{\alpha \beta}=\overline{\boldsymbol{g}}_{\alpha} \cdot \overline{\boldsymbol{g}}_{\beta}
$$

where $\bar{g}_{\alpha \beta}$ are the coordinates of the interface spatial metric tensor. It bears emphasis that the Helmholtz energy of the form $\bar{\psi}=\bar{\psi}\left(\bar{g}_{\alpha \beta}\right)$ is indeed frame indifferent but not automatically covariant. This shall be compared with the Helmholtz energy of the form $\bar{\psi}=\bar{\psi}(\overline{\boldsymbol{C}})$ that is covariant implying a stronger material frame indifference.

\subsection{Consequences implied by the interface elasticity theory}

This section collates some significant consequences following from the interface elasticity theory. Some of the consequences are intuitive and well-established while others remain elusive and require detailed discussion.

$\overline{\boldsymbol{F}}$ is both superficial and tangential. The interface deformation gradient $\overline{\boldsymbol{F}}$ is superficial by definition since $\overline{\boldsymbol{F}} \cdot \overline{\boldsymbol{N}}=\mathbf{0}$. Due to the particular structure of $\overline{\boldsymbol{F}}$ given by Eq. $(2$, it is also tangential since $\overline{\boldsymbol{n}} \cdot \overline{\boldsymbol{F}}=\mathbf{0}$. Consequently, $\overline{\boldsymbol{F}}$ is superficial with respect to the material configuration and tangential with respect to the spatial configuration.

By definition, the interface Piola stress $\bar{P}$ is superficial, but not necessarily tangential. Following the Cauchy theorem for a first-order continuum theory, it becomes evident that a fundamental property of the interface elasticity theory is the superficiality of the interface Piola stress in the sense that $\overline{\boldsymbol{P}} \cdot \overline{\boldsymbol{N}}=\mathbf{0}$. Nevertheless, stresses $\overline{\boldsymbol{P}}$ and $\boldsymbol{P}$ have different dimensions and refer to different phenomena and hence, cannot be related, in general. Somewhat surprisingly, the rate of the interface deformation gradient is only superficial but not necessarily tangential or $\dot{\overline{\boldsymbol{F}}} \cdot \overline{\boldsymbol{N}}=\mathbf{0}$ but $\overline{\boldsymbol{n}} \cdot \dot{\overline{\boldsymbol{F}}} \neq \mathbf{0}$. Therefore, the format of the interface external power $\overline{\mathcal{P}}_{\text {ex }}=\overline{\boldsymbol{P}}: \dot{\overline{\boldsymbol{F}}}$ suggests the interface stress $\overline{\boldsymbol{P}}$ to span the same space as $\dot{\overline{\boldsymbol{F}}}$ hence, not necessarily tangential by definition, see Eq. (11) 2 . 
Interface Piola stress $\overline{\boldsymbol{P}}$ is tangential due to the angular momentum balance. Central to the interface elasticity theory are superficiality properties of $\overline{\boldsymbol{F}}$ and $\overline{\boldsymbol{P}}$. While $\overline{\boldsymbol{F}}$ is also tangential, we cannot at this state claim the same for $\overline{\boldsymbol{P}}$. However, balance of angular momentum on the interface $\left.{ }_{10}\right]_{2}$ requires $\overline{\boldsymbol{P}}$ to be tangential as

$$
\left.\begin{array}{ll}
\text { by definition: } & \overline{\boldsymbol{n}} \cdot \overline{\boldsymbol{F}}=\mathbf{0} \\
\text { ang. mom. bal.: } & \overline{\boldsymbol{P}} \cdot \overline{\boldsymbol{F}}^{\mathrm{t}}=\overline{\boldsymbol{F}} \cdot \overline{\boldsymbol{P}}^{\mathrm{t}}
\end{array}\right\} \Rightarrow \overline{\boldsymbol{n}} \cdot \overline{\boldsymbol{P}} \cdot \overline{\boldsymbol{F}}^{\mathrm{t}}=\underbrace{\overline{\boldsymbol{n}} \cdot \overline{\boldsymbol{F}}}_{=\mathbf{0}} \cdot \overline{\boldsymbol{P}}^{\mathrm{t}} \Rightarrow \overline{\boldsymbol{n}} \cdot \overline{\boldsymbol{P}}=\mathbf{0} .
$$

With these results, $\overline{\boldsymbol{P}}$ is superficial with respect to the material configuration but tangential with respect to the spatial configuration. In short, both the interface deformation gradient $\overline{\boldsymbol{F}}$ and the interface Piola stress $\overline{\boldsymbol{P}}$ are superficial as well as tangential. Thus, the interface stress vectors and interface stress tensors are related according to

$$
\overline{\boldsymbol{P}}=\overline{\boldsymbol{p}}^{\alpha} \otimes \overline{\boldsymbol{G}}_{\alpha} \quad \Leftrightarrow \quad \overline{\boldsymbol{p}}^{\alpha}=\bar{P}^{\beta \alpha} \overline{\boldsymbol{g}}_{\beta} \quad \text { with } \quad \alpha, \beta \in\{1,2\},
$$

which shall be compared with Eq. 13 . While $\overline{\boldsymbol{F}}$ is tangential by definition, $\overline{\boldsymbol{P}}$ is tangential because of the balance of angular momentum. This observation is particularly important since it rules out the existence of the interface shear detailed next.

Dependence of energy on the interface normal $\bar{n}$ leads to the notion of interface shear. As previously discussed, the interface Helmholtz energy density $\bar{\psi}$ shall be a function of the interface deformation gradient $\overline{\boldsymbol{F}}$ or equivalently a function of $\overline{\boldsymbol{g}}_{\alpha}$. Since the interface normal $\overline{\boldsymbol{n}}$ depends on the interface deformation gradient $\overline{\boldsymbol{F}}$ and can be constructed via the outer product of the interface base vectors, we may allow the interface Helmholtz energy to depend explicitly on $\overline{\boldsymbol{n}}$ as $\bar{\psi}=\bar{\psi}(\overline{\boldsymbol{F}}, \overline{\boldsymbol{n}})$. Using relation $\partial \overline{\boldsymbol{n}} / \partial \overline{\boldsymbol{F}}=-\overline{\boldsymbol{n}} \bar{\otimes} \overline{\boldsymbol{F}}^{\text {-t }}$ in index notation $\left[\overline{\boldsymbol{n}} \bar{\otimes} \overline{\boldsymbol{F}}^{\text {-t }}\right]_{i j k}=[\overline{\boldsymbol{n}}]_{j}\left[\overline{\boldsymbol{F}}^{\text {-t }}\right]_{i k}$, proven in Appendix B, we expand the interface stress

$$
\overline{\boldsymbol{P}}=\frac{\partial \bar{\psi}}{\partial \overline{\boldsymbol{F}}}=\left.\frac{\partial \bar{\psi}}{\partial \overline{\boldsymbol{F}}}\right|_{\overline{\boldsymbol{n}}}+\frac{\partial \bar{\psi}}{\partial \overline{\boldsymbol{n}}} \cdot \frac{\partial \overline{\boldsymbol{n}}}{\partial \overline{\boldsymbol{F}}}=\left.\frac{\partial \bar{\psi}}{\partial \overline{\boldsymbol{F}}}\right|_{\overline{\boldsymbol{n}}}-\overline{\boldsymbol{n}} \otimes \overline{\boldsymbol{\Gamma}} \quad \text { with } \quad \overline{\boldsymbol{\Gamma}}=\frac{\partial \bar{\psi}}{\partial \overline{\boldsymbol{n}}} \cdot \overline{\boldsymbol{F}}^{-\mathrm{t}},
$$

in which $\overline{\boldsymbol{\Gamma}}$ is a vector tangential to the interface on the material configuration. At first glance, the structure of $\overline{\boldsymbol{n}} \otimes \overline{\boldsymbol{\Gamma}}$ implies that the interface stress contains a normal component in the spatial configuration and ties to the surface shear concept [69 71]. However, due to the interface balance of angular momentum, the interface stress needs to be tangential and satisfy $\overline{\boldsymbol{n}} \cdot \overline{\boldsymbol{P}}=\mathbf{0}$. Therefore,

$$
\overline{\boldsymbol{n}} \cdot \overline{\boldsymbol{P}}=\left.\mathbf{0} \quad \Rightarrow \quad \overline{\boldsymbol{n}} \cdot \frac{\partial \bar{\psi}}{\partial \overline{\boldsymbol{F}}}\right|_{\overline{\boldsymbol{n}}}-\overline{\boldsymbol{\Gamma}}=\mathbf{0} \quad \Rightarrow \quad \overline{\boldsymbol{\Gamma}}=\left.\overline{\boldsymbol{n}} \cdot \frac{\partial \bar{\psi}}{\partial \overline{\boldsymbol{F}}}\right|_{\overline{\boldsymbol{n}}},
$$

which essentially states that $\partial \bar{\psi} /\left.\partial \overline{\boldsymbol{F}}\right|_{\overline{\boldsymbol{n}}}$ does contain a non-tangential term but overall the surface shear contribution cancels from the interface stress $\overline{\boldsymbol{P}}$. The interface stress $\overline{\boldsymbol{P}}$ reads

$$
\overline{\boldsymbol{P}}=\left.\frac{\partial \bar{\psi}}{\partial \overline{\boldsymbol{F}}}\right|_{\overline{\boldsymbol{n}}}-\overline{\boldsymbol{n}} \otimes \overline{\boldsymbol{\Gamma}}=\left.\frac{\partial \bar{\psi}}{\partial \overline{\boldsymbol{F}}}\right|_{\overline{\boldsymbol{n}}}-\left.\overline{\boldsymbol{n}} \otimes \overline{\boldsymbol{n}} \cdot \frac{\partial \bar{\psi}}{\partial \overline{\boldsymbol{F}}}\right|_{\overline{\boldsymbol{n}}}=\left.[\boldsymbol{i}-\overline{\boldsymbol{n}} \otimes \overline{\boldsymbol{n}}] \cdot \frac{\partial \bar{\psi}}{\partial \overline{\boldsymbol{F}}}\right|_{\overline{\boldsymbol{n}}}=\left.\overline{\boldsymbol{i}} \cdot \frac{\partial \bar{\psi}}{\partial \overline{\boldsymbol{F}}}\right|_{\overline{\boldsymbol{n}}},
$$


which clearly reveals the projection via the interface identity on the spatial configuration from left. As we will see next, the interface Helmholtz energy density $\bar{\psi}$ cannot depend on the interface normal, thereby excluding the interface shear a priori.

Note, that this manuscript and consequently the aforementioned discussions are particularly relevant to material interfaces and deformational mechanics. The surface shear may exist in configurational mechanics [72 [75] or for evolving interfaces [76], see also [77. For deformational mechanics and non-evolving coherent interfaces though, the interface shear is not admissible at all.

Material frame indifference rules out the dependence of $\bar{\psi}$ on $\bar{n}$. In the following, we prove that the normal vector cannot enter the energy. In doing so, we start from the Helmholtz energy $\bar{\psi}=\bar{\psi}(\overline{\boldsymbol{F}}, \overline{\boldsymbol{n}})$ explicitly accounting for the interface unit normal $\overline{\boldsymbol{n}}$. Material frame indifference requires the energy $\bar{\psi}$ to be invariant with respect to rotations as

$$
\bar{\psi}=\bar{\psi}(\overline{\boldsymbol{F}}, \overline{\boldsymbol{n}})=\bar{\psi}(\boldsymbol{Q} \cdot \overline{\boldsymbol{F}}, \boldsymbol{Q} \cdot \overline{\boldsymbol{n}}) \quad \forall \boldsymbol{Q} \in \mathcal{S O}(3)
$$

The objectivity requirement 29 holds if (i) $\overline{\boldsymbol{F}}$ enters the energy via $\overline{\boldsymbol{C}}:=\overline{\boldsymbol{F}}^{\mathrm{t}} \cdot \overline{\boldsymbol{F}}$ and if (ii) $\overline{\boldsymbol{n}}$ enters the energy via $\overline{\boldsymbol{n}} \cdot \overline{\boldsymbol{n}}$ or $\overline{\boldsymbol{n}} \cdot \overline{\boldsymbol{F}}$. Consequently,

$$
\bar{\psi}=\bar{\psi}(\overline{\boldsymbol{F}}, \overline{\boldsymbol{n}})=\bar{\psi}(\overline{\boldsymbol{C}}, \underbrace{\overline{\boldsymbol{n}} \cdot \overline{\boldsymbol{F}}}_{=0}, \underbrace{\overline{\boldsymbol{n}} \cdot \overline{\boldsymbol{n}}}_{=1}) \quad \Rightarrow \quad \bar{\psi}=\bar{\psi}(\overline{\boldsymbol{C}})
$$

in which we have used that $\overline{\boldsymbol{F}}$ is tangential.

Material frame indifference implies balance of angular momentum. Enforcing material frame indifference of the Helmholtz energy, requires the interface deformation gradient to enter the energy through $\bar{C}$. Therefore, the interface Piola stress reads

$$
\overline{\boldsymbol{P}}=\frac{\partial \bar{\psi}}{\partial \overline{\boldsymbol{F}}}=\frac{\partial \bar{\psi}}{\partial \overline{\boldsymbol{C}}}: \frac{\partial \overline{\boldsymbol{C}}}{\partial \overline{\boldsymbol{F}}}=\overline{\boldsymbol{F}} \cdot \overline{\boldsymbol{S}} \quad \text { with } \quad \overline{\boldsymbol{S}}:=2 \frac{\partial \bar{\psi}}{\partial \overline{\boldsymbol{C}}},
$$

whereby $\overline{\boldsymbol{S}}$ denotes the symmetric interface Piola-Kirchhoff stress. An important consequence of the relation $\overline{\boldsymbol{P}}=\overline{\boldsymbol{F}} \cdot \overline{\boldsymbol{S}}$ is that

$$
\overline{\boldsymbol{P}} \cdot \overline{\boldsymbol{F}}^{\mathrm{t}}=\overline{\boldsymbol{F}} \cdot \overline{\boldsymbol{S}} \cdot \overline{\boldsymbol{F}}^{\mathrm{t}}=\overline{\boldsymbol{F}} \cdot[\overline{\boldsymbol{F}} \cdot \overline{\boldsymbol{S}}]^{\mathrm{t}}=\overline{\boldsymbol{F}} \cdot \overline{\boldsymbol{P}}^{\mathrm{t}}
$$

and therefore, balance of angular momentum on the interface is satisfied a priori. Furthermore, note that the relation $\overline{\boldsymbol{P}}=\overline{\boldsymbol{F}} \cdot \overline{\boldsymbol{S}}$ automatically furnishes a tangential as well as superficial interface stress $\overline{\boldsymbol{P}}$.

Isotropic interface Helmholtz energy is expressed in terms of the two invariants of $\bar{C}$. Following the representation theorem for isotropic functions, the interface Helmholtz energy $\bar{\psi}(\overline{\boldsymbol{C}})$ for isotropic interface behavior shall be expressed as $\bar{\psi}\left(\bar{I}_{1}, \bar{I}_{2}\right)$ with $\bar{I}_{1}=\overline{\boldsymbol{C}}: \overline{\boldsymbol{I}}$ and $\bar{I}_{2}=\overline{\operatorname{Det}} \overline{\boldsymbol{C}}$ being the invariants of $\overline{\boldsymbol{C}}$. An 
interesting consequence of isotropic interface response is that the interface stresses, without loss of generality, simplify to

$$
\begin{aligned}
\overline{\boldsymbol{S}}=2 \frac{\partial \bar{\psi}}{\partial \bar{I}_{1}} \overline{\boldsymbol{I}}+2 \frac{\partial \bar{\psi}}{\partial \bar{I}_{2}} \bar{I}_{2} \overline{\boldsymbol{C}}^{-1} & \text { or } & \overline{\boldsymbol{S}} & =2 \frac{\partial \bar{\psi}}{\partial \bar{I}_{1}} \overline{\boldsymbol{G}}_{\alpha} \otimes \overline{\boldsymbol{G}}^{\alpha}+2 \frac{\partial \bar{\psi}}{\partial \bar{I}_{2}} \bar{I}_{2} \bar{g}^{\alpha \beta} \overline{\boldsymbol{G}}_{\alpha} \otimes \overline{\boldsymbol{G}}_{\beta}, \\
\overline{\boldsymbol{P}}=2 \frac{\partial \bar{\psi}}{\partial \bar{I}_{1}} \overline{\boldsymbol{F}}+2 \frac{\partial \bar{\psi}}{\partial \bar{I}_{2}} \bar{I}_{2} \overline{\boldsymbol{F}}^{\text {- }} & \text { or } & \overline{\boldsymbol{P}} & =2 \frac{\partial \bar{\psi}}{\partial \bar{I}_{1}} \overline{\boldsymbol{g}}_{\alpha} \otimes \overline{\boldsymbol{G}}^{\alpha}+2 \frac{\partial \bar{\psi}}{\partial \bar{I}_{2}} \bar{I}_{2} \overline{\boldsymbol{g}}^{\alpha} \otimes \overline{\boldsymbol{G}}_{\alpha},
\end{aligned}
$$

which clearly indicate the structures of the interface stresses. Note that $\overline{\boldsymbol{S}}$ is both superficial and tangential in the material configuration while $\overline{\boldsymbol{P}}$ is tangential in the spatial configuration and superficial in the material configuration. Instead of $\bar{I}_{1}$ and $\bar{I}_{2}$, we could choose any set of two independent invariants of $\bar{C}$ such as $\overline{\boldsymbol{C}}: \overline{\boldsymbol{I}}$ and $\overline{\boldsymbol{C}}^{2}: \overline{\boldsymbol{I}}$. Nevertheless, the resultant expressions for $\overline{\boldsymbol{P}}$ and $\overline{\boldsymbol{S}}$ would be formally identical regardless of the choice of the invariants.

Table 1 summarizes the geometrically exact interface elasticity theory and fundamental concepts associated to the theory. Furthermore, the governing equations are cast into the classic format [6, 7, 36, among others] and the alternative format in accordance with 68 . 
differential geometry of interfaces (corresponding to the material configuration)

$\begin{array}{ll}\text { co-variant and contra-variant bases } & \overline{\boldsymbol{G}}_{\alpha}, \overline{\boldsymbol{G}}^{\alpha} \\ \text { normal } & \overline{\boldsymbol{N}}= \pm \overline{\boldsymbol{G}}_{1} \times \overline{\boldsymbol{G}}_{2} /\left|\overline{\boldsymbol{G}}_{1} \times \overline{\boldsymbol{G}}_{2}\right| \\ \text { gradient operator } & \overline{\operatorname{Grad}}\left\{\bullet=\partial\{\bullet\} / \Theta^{\alpha} \otimes \overline{\boldsymbol{G}}^{\alpha}\right. \\ \text { divergence operator } & \overline{\operatorname{Div}}\{\bullet\}=\partial\left\{\bullet / \Theta^{\alpha} \cdot \overline{\boldsymbol{G}}^{\alpha}\right. \\ \text { identity } & \overline{\mathbf{I}}=\overline{\boldsymbol{G}}_{\alpha} \otimes \overline{\boldsymbol{G}}^{\alpha}=\boldsymbol{I}-\overline{\boldsymbol{N}} \otimes \overline{\boldsymbol{N}}\end{array}$

kinematics of interfaces

material and spatial coordinates

$\overline{\boldsymbol{X}}, \overline{\boldsymbol{x}}$

non-linear map

$\bar{\varphi} \quad$ with $\quad \bar{x}=\bar{\varphi}(\bar{X})$

linear tangent map

$\overline{\boldsymbol{F}} \quad$ with $\quad \mathrm{d} \overline{\boldsymbol{x}}=\overline{\boldsymbol{F}} \cdot \mathrm{d} \overline{\boldsymbol{X}}$

linear normal map

$\overline{\operatorname{Cof}} \overline{\boldsymbol{F}} \quad$ with $\quad \mathrm{d} \overline{\boldsymbol{l}}=\overline{\operatorname{Cof}} \overline{\boldsymbol{F}} \cdot \mathrm{d} \overline{\boldsymbol{L}}$

determinant operator

$\overline{\operatorname{Det}} \overline{\boldsymbol{F}}=\left|\overline{\boldsymbol{F}} \cdot \overline{\boldsymbol{G}}_{1} \times \overline{\boldsymbol{F}} \cdot \overline{\boldsymbol{G}}_{2}\right| /\left|\overline{\boldsymbol{G}}_{1} \times \overline{\boldsymbol{G}}_{2}\right|$

governing equations of interfaces

classic format

lin. mom. balance

ang. mom. balance

external power

deformation rate

stress measure

dissipation inequality

constitutive law

objective energy

$$
\begin{aligned}
& \overline{\operatorname{Div}} \overline{\boldsymbol{P}}+\llbracket \boldsymbol{P} \rrbracket \cdot \overline{\boldsymbol{N}}=\mathbf{0} \\
& \boldsymbol{\varepsilon}:\left[\overline{\boldsymbol{F}} \cdot \overline{\boldsymbol{P}}^{\mathrm{t}}\right]=\mathbf{0} \\
& \overline{\mathcal{P}}_{\mathrm{ex}}=\overline{\boldsymbol{P}}: \dot{\overline{\boldsymbol{F}}} \\
& \dot{\overline{\boldsymbol{F}}}=\dot{\overline{\boldsymbol{g}}}_{\alpha} \otimes \overline{\boldsymbol{G}}^{\alpha} \\
& \overline{\boldsymbol{P}}=\overline{\boldsymbol{p}}^{\alpha} \otimes \overline{\boldsymbol{G}}_{\alpha} \\
& \overline{\mathcal{D}}=\overline{\boldsymbol{P}}: \dot{\overline{\boldsymbol{F}}}-\dot{\bar{\psi}} \geq 0 \\
& \overline{\boldsymbol{P}}=\partial \bar{\psi} / \partial \overline{\boldsymbol{F}} \\
& \bar{\psi}=\bar{\psi}(\overline{\boldsymbol{C}})
\end{aligned}
$$

alternative format

$$
\begin{aligned}
& \overline{\operatorname{Grad}} \overline{\boldsymbol{p}}^{\alpha} \cdot \overline{\boldsymbol{G}}_{\alpha}+\llbracket \boldsymbol{p}^{i} \otimes \boldsymbol{G}_{i} \rrbracket \cdot \overline{\boldsymbol{N}}=\mathbf{0} \\
& \boldsymbol{\varepsilon}:\left[\overline{\boldsymbol{g}}_{\alpha} \otimes \overline{\boldsymbol{p}}^{\alpha}\right]=\mathbf{0} \\
& \overline{\mathcal{P}}_{\mathrm{ex}}=\overline{\boldsymbol{p}}^{\alpha} \cdot \dot{\overline{\boldsymbol{g}}}_{\alpha} \\
& \dot{\overline{\boldsymbol{g}}}_{\alpha}=\dot{\overline{\boldsymbol{F}}} \cdot \overline{\boldsymbol{G}}_{\alpha} \\
& \overline{\boldsymbol{p}}^{\alpha}=\bar{P}^{\beta \alpha} \overline{\boldsymbol{g}}_{\beta} \\
& \overline{\mathcal{D}}=\overline{\boldsymbol{p}}^{\alpha} \cdot \dot{\overline{\boldsymbol{g}}}_{\alpha}-\dot{\bar{\psi}} \geq 0 \\
& \overline{\boldsymbol{p}}^{\alpha}=\partial \bar{\psi} / \partial \overline{\boldsymbol{g}}_{\alpha} \\
& \bar{\psi}=\bar{\psi}\left(\bar{g}_{\alpha \beta}\right)
\end{aligned}
$$

Table 1: Summary of interface elasticity theory. The interface unit normal $\bar{N}$ points from the minus to the plus side of the interface and carries by definition a \pm sign to indicate that this formulation cannot determine the direction of the normal and that shall be constrained with the surrounding bulk. The stress measures are energetically conjugate to the deformation rate measures. The corresponding index for bulk quantities is denoted $i \in\{1,2,3\}$ to distinguish from the one associated to the interface quantities with $\alpha \in\{1,2\}$. 


\section{Linearized interface elasticity theory}

So far, we have introduced a geometrically exact interface elasticity theory at finite deformations together with its natural consequences. However, many applications of the interface elasticity theory deal with the behavior of materials not only at small scales but also at small strains. Therefore, it is extremely useful to derive a consistently linearized interface elasticity theory, see [78] among others. Linearization means that expressions for stresses and strains depend linearly on the displacements; essentially, this implies that an exact non-linear relation is replaced by its tangent at the point in question. Obviously, a linearized theory is meaningful if the displacement gradients are small, i.e. $\|\overline{\operatorname{Grad}} \overline{\boldsymbol{u}}\|<\delta$ with sufficiently small $\delta \ll 1$.

We limit the linearization procedure to the interface as the corresponding derivations for the bulk are standard and well-established. To proceed, we define the linearization operator $\mathscr{L}$ on the interface as

$$
\mathscr{L}\{\bullet\}=\left.\{\bullet\}\right|_{\boldsymbol{I}}+\left.\frac{\partial\{\bullet\}}{\partial \overline{\boldsymbol{F}}}\right|_{\boldsymbol{I}}:[\overline{\boldsymbol{F}}-\overline{\boldsymbol{I}}]=\left.\{\bullet\}\right|_{\boldsymbol{I}}+\left.\frac{\partial\{\bullet\}}{\partial \overline{\boldsymbol{F}}}\right|_{\boldsymbol{I}}: \overline{\operatorname{Grad}} \overline{\boldsymbol{u}},
$$

with $\overline{\boldsymbol{u}}$ being the infinitesimal displacement on the interface, but not necessarily tangential to the interface. From definition (34), it follows instantly that

$$
\mathscr{L} \overline{\boldsymbol{F}}=\overline{\boldsymbol{I}}+\overline{\mathrm{Grad}} \overline{\boldsymbol{u}},
$$

which takes an analogous format in the bulk. However, the linearized interface right Cauchy-Green tensor $\bar{C}$ has some small but important differences from its bulk counterpart. Linearization of $\bar{C}$ reads

$$
\mathscr{L} \overline{\boldsymbol{C}}=\overline{\boldsymbol{I}}+2 \overline{\mathbb{I}}^{\mathrm{sym}}: \overline{\operatorname{Grad}} \overline{\boldsymbol{u}} \quad \text { with } \quad \overline{\mathbb{I}}^{\text {sym }}:=\left.\frac{1}{2} \frac{\partial \overline{\boldsymbol{C}}}{\partial \overline{\boldsymbol{F}}}\right|_{\boldsymbol{I}}=\frac{1}{2}\left[\overline{\boldsymbol{G}}^{\beta} \otimes \overline{\boldsymbol{G}}^{\alpha}+\overline{\boldsymbol{G}}^{\alpha} \otimes \overline{\boldsymbol{G}}^{\beta}\right] \otimes \overline{\boldsymbol{G}}^{\alpha} \otimes \overline{\boldsymbol{G}}_{\beta} .
$$

It appears that $\overline{\mathbb{I}}^{\text {sym }}$ has the property that, for any second-order tensor $\overline{\boldsymbol{A}}$, we have $\overline{\mathbb{I}}^{\text {sym }}: \overline{\boldsymbol{A}}=\overline{\mathbb{I}}^{\text {sym }}: \overline{\boldsymbol{A}}^{\text {sym }}$. It is important to note that $\overline{\mathbb{I}}^{\text {sym }}$ not only does symmetrize the second-order tensor $\overline{\operatorname{Grad}} \overline{\boldsymbol{u}}$, but that it functions as a projection to the reference configuration, see [79] for further details in the bulk. Expression (36) can be written as

$$
\mathscr{L} \overline{\boldsymbol{C}}=\overline{\boldsymbol{I}}+\overline{\boldsymbol{I}} \cdot \overline{\operatorname{Grad}} \overline{\boldsymbol{u}}+[\overline{\operatorname{Grad}} \overline{\boldsymbol{u}}]^{\mathrm{t}} \cdot \overline{\boldsymbol{I}} \neq \overline{\boldsymbol{I}}+2[\overline{\mathrm{Grad}} \overline{\boldsymbol{u}}]^{\mathrm{sym}},
$$

which is different from its bulk counterpart. In fact, the symmetric interface displacement gradient

$$
[\overline{\operatorname{Grad}} \overline{\boldsymbol{u}}]^{\mathrm{sym}}=\frac{1}{2}\left[\overline{\operatorname{Grad}} \overline{\boldsymbol{u}}+[\overline{\operatorname{Grad}} \overline{\boldsymbol{u}}]^{\mathrm{t}}\right],
$$

is not necessarily tangential or superficial to the interface and, hence, cannot be a suitable interface strain measure for linear elasticity. Instead, we define the linearized interface strain $\bar{\epsilon}$ as

$$
\overline{\boldsymbol{\epsilon}}=\frac{1}{2}\left[\overline{\boldsymbol{I}} \cdot \overline{\operatorname{Grad}} \overline{\boldsymbol{u}}+[\overline{\mathrm{Grad}} \overline{\boldsymbol{u}}]^{\mathrm{t}} \cdot \overline{\boldsymbol{I}}\right]=\overline{\mathbb{I}}^{\mathrm{sym}}: \overline{\mathrm{Grad}} \overline{\boldsymbol{u}}=\overline{\mathbb{I}}^{\mathrm{sym}}:[\overline{\mathrm{Grad}} \overline{\boldsymbol{u}}]^{\mathrm{sym}}
$$

In this case, the linearized strain $\overline{\boldsymbol{\epsilon}}$ relates to $\bar{C}$ through

$$
\mathscr{L} \overline{\boldsymbol{C}}=\overline{\boldsymbol{I}}+2 \overline{\boldsymbol{\epsilon}}
$$


Next, we apply the linearization procedure on the governing equations, namely (i) the balance of linear momentum, (ii) the balance of angular momentum and (iii) the constitutive laws.

\section{Linearized balance of linear momentum}

Linearizing the balance of linear momentum on the interface at finite deformation $(10)_{1}$ reads

$$
\overline{\operatorname{Div}} \overline{\boldsymbol{P}}+\llbracket \boldsymbol{P} \rrbracket \cdot \overline{\boldsymbol{N}}=\mathbf{0} \quad \stackrel{\mathscr{L}}{\Longrightarrow} \quad \overline{\operatorname{Div}} \overline{\boldsymbol{\Pi}}+\llbracket \boldsymbol{\Pi} \rrbracket \cdot \overline{\boldsymbol{N}}=\mathbf{0},
$$

in which $\Pi$ and $\bar{\Pi}$ are the linear stresses in the bulk and on the interface, respectively. Bulk stress $\Pi$ is standard and we only elaborate on the interface stress $\bar{\Pi}$ as

$$
\begin{aligned}
\overline{\boldsymbol{\Pi}}=\mathscr{L} \overline{\boldsymbol{P}}=\mathscr{L}(\overline{\boldsymbol{F}} \cdot \overline{\boldsymbol{S}}) & =\left.[\overline{\boldsymbol{F}} \cdot \overline{\boldsymbol{S}}]\right|_{\boldsymbol{I}}+\left.\frac{\partial(\overline{\boldsymbol{F}} \cdot \overline{\boldsymbol{S}})}{\partial \overline{\boldsymbol{F}}}\right|_{\boldsymbol{I}}: \overline{\operatorname{Grad}} \overline{\boldsymbol{u}} \\
& =\overline{\boldsymbol{\Pi}}_{0}+\left[\overline{\mathbb{C}}: \overline{\mathbb{I}}^{\mathrm{sym}}+\boldsymbol{I} \bar{\otimes} \overline{\boldsymbol{\Pi}}_{0}\right]: \overline{\mathrm{Grad}} \overline{\boldsymbol{u}}
\end{aligned}
$$

where

$$
\overline{\boldsymbol{\Pi}}_{0}:=\left.\overline{\boldsymbol{S}}\right|_{\boldsymbol{I}}=\left.2 \frac{\partial \bar{\psi}}{\partial \overline{\boldsymbol{C}}}\right|_{\boldsymbol{I}} \quad \text { and } \quad \overline{\mathbb{C}}:=\left.2 \frac{\partial \overline{\boldsymbol{S}}}{\partial \overline{\boldsymbol{C}}}\right|_{\boldsymbol{I}}=\left.4 \frac{\partial^{2} \bar{\psi}}{\partial \overline{\boldsymbol{C}} \partial \overline{\boldsymbol{C}}}\right|_{\boldsymbol{I}} .
$$

It appears that the initial stress $\overline{\boldsymbol{\Pi}}_{0}$ is symmetric as it derives from $\bar{\psi}(\overline{\boldsymbol{C}})$. Moreover, the linear interface stress $\bar{\Pi}$ may also be written as

$$
\overline{\boldsymbol{\Pi}}=\overline{\boldsymbol{\Pi}}_{0}+\overline{\mathbb{C}}: \overline{\boldsymbol{\epsilon}}+\overline{\operatorname{Grad}} \overline{\boldsymbol{u}} \cdot \overline{\boldsymbol{\Pi}}_{0} .
$$

We observe that the last term, i.e. $\overline{\operatorname{Grad}} \overline{\boldsymbol{u}} \cdot \overline{\boldsymbol{\Pi}}_{0}$, is a result of a differentiation of $\overline{\boldsymbol{F}}$ in the expression $\overline{\boldsymbol{P}}=\overline{\boldsymbol{F}} \cdot \overline{\boldsymbol{S}}$, i.e. this term is related to the change of kinematics and not to the change of Helmholtz energy. Finally, with (44), the linearized balance of linear momentum reads

$$
\overline{\operatorname{Div}}\left(\overline{\boldsymbol{\Pi}}_{0}+\overline{\mathbb{C}}: \overline{\boldsymbol{\epsilon}}+\overline{\operatorname{Grad}} \overline{\boldsymbol{u}} \cdot \overline{\boldsymbol{\Pi}}_{0}\right)+\llbracket \boldsymbol{\Pi} \rrbracket \cdot \overline{\boldsymbol{N}}=\mathbf{0},
$$

subject to the boundary condition

$$
\left[\overline{\boldsymbol{\Pi}}_{0}+\overline{\mathbb{C}}: \overline{\boldsymbol{\epsilon}}+\overline{\operatorname{Grad}} \overline{\boldsymbol{u}} \cdot \overline{\boldsymbol{\Pi}}_{0}\right] \cdot \widetilde{\boldsymbol{N}}=\widetilde{\boldsymbol{b}}_{0} .
$$

The format of Eq. (44) indicates that $\overline{\boldsymbol{\Pi}}$ is non-symmetric, in general, due to the controversial term $\overline{\operatorname{Grad}} \overline{\boldsymbol{u}}$, see [43, 80, 89] among others.

Let $\boldsymbol{\Pi}_{0}$ denote the initial stresses in the bulk. Then, the linearized balance of linear momentum (45) at the reference configuration, i.e. $\overline{\boldsymbol{F}}=\overline{\boldsymbol{I}}$, reads

$$
\overline{\operatorname{Div}} \bar{\Pi}_{0}+\llbracket \boldsymbol{\Pi}_{0} \rrbracket \cdot \overline{\boldsymbol{N}}=\mathbf{0} .
$$

Inserting Eq. 477) into Eq. (45) yields

$$
\overline{\operatorname{Div}}\left(\overline{\mathbb{C}}: \overline{\boldsymbol{\epsilon}}+\overline{\operatorname{Grad}} \overline{\boldsymbol{u}} \cdot \overline{\boldsymbol{\Pi}}_{0}\right)+\llbracket \boldsymbol{\Pi}-\boldsymbol{\Pi}_{0} \rrbracket \cdot \overline{\boldsymbol{N}}=\mathbf{0} .
$$


Eq. (48) clearly shows that the controversial non-symmetric term $\overline{\operatorname{Grad}} \overline{\boldsymbol{u}} \cdot \overline{\boldsymbol{\Pi}}_{0}$ may play a significant role even for an infinitesimal displacement gradient since its influence depends on the magnitude of the initial stress $\overline{\boldsymbol{\Pi}}_{0}$. Furthermore, the term $\overline{\text { Grad }} \overline{\boldsymbol{u}}$ itself is of the same order as $\overline{\boldsymbol{\epsilon}}$. Finally, in the presence of initial stresses, the linearization of a bulk model also leads to non-symmetric stresses.

\section{Linearized balance of angular momentum}

In order to obtain the linearized balance of angular momentum, we apply the identity

$$
\mathscr{L}(\boldsymbol{A} \cdot \boldsymbol{B})=\left.\mathscr{L} \boldsymbol{A} \cdot \boldsymbol{B}\right|_{\boldsymbol{I}}+\left.\boldsymbol{A}\right|_{\boldsymbol{I}} \cdot \mathscr{L} \boldsymbol{B}-\left.(\boldsymbol{A} \cdot \boldsymbol{B})\right|_{\boldsymbol{I}}
$$

on the interface angular momentum balance 10$]_{2}$. Therefore

$$
\begin{aligned}
\overline{\boldsymbol{P}} \cdot \overline{\boldsymbol{F}}^{\mathrm{t}} & =\overline{\boldsymbol{F}} \cdot \overline{\boldsymbol{P}}^{\mathrm{t}} \\
& \left.\stackrel{\mathscr{L}}{\Longrightarrow} \quad \mathscr{L} \overline{\boldsymbol{P}} \cdot \overline{\boldsymbol{F}^{\mathrm{t}}}\right|_{\boldsymbol{I}}+\left.\overline{\boldsymbol{P}}\right|_{\boldsymbol{I}} \cdot \mathscr{L} \overline{\boldsymbol{F}}^{\mathrm{t}}-\left.\left(\overline{\boldsymbol{P}} \cdot \overline{\boldsymbol{F}}^{\mathrm{t}}\right)\right|_{\boldsymbol{I}}=\left.\mathscr{L} \overline{\boldsymbol{F}} \cdot \overline{\boldsymbol{P}}^{\mathrm{t}}\right|_{\boldsymbol{I}}+\left.\overline{\boldsymbol{F}}\right|_{\boldsymbol{I}} \cdot \mathscr{L} \overline{\boldsymbol{P}}^{\mathrm{t}}-\left.(\overline{\boldsymbol{F}} \cdot \overline{\boldsymbol{P}} \mathrm{t})\right|_{\boldsymbol{I}} .
\end{aligned}
$$

Using $\left.\overline{\boldsymbol{F}}\right|_{\boldsymbol{I}}=\overline{\boldsymbol{I}},\left.\overline{\boldsymbol{P}}\right|_{\boldsymbol{I}}=\overline{\boldsymbol{\Pi}}_{0}$ and the symmetry property of $\overline{\boldsymbol{\Pi}}_{0}$ we obtain

$$
\overline{\boldsymbol{\Pi}} \cdot \overline{\boldsymbol{I}}+\overline{\boldsymbol{\Pi}}_{0} \cdot[\overline{\boldsymbol{I}}+\overline{\operatorname{Grad}} \overline{\boldsymbol{u}}]^{\mathrm{t}}=[\overline{\boldsymbol{I}}+\overline{\operatorname{Grad}} \overline{\boldsymbol{u}}] \cdot \overline{\boldsymbol{\Pi}}_{0}+\overline{\boldsymbol{I}} \cdot \overline{\boldsymbol{\Pi}}^{\mathrm{t}} .
$$

Inserting the linearized interface stress $\bar{\Pi}$ from Eq. 44. into the expression above shows that Eq. 51. is trivially fulfilled. Thus, the linearized balance of angular momentum on the interface is satisfied a priori.

\section{Linearized constitutive laws}

Let us first identify an expression for the linearized stress $\mathscr{L} \overline{\boldsymbol{S}}$ of $\overline{\boldsymbol{S}}$. It follows from (34) that

$$
\overline{\boldsymbol{\Sigma}}:=\mathscr{L} \overline{\boldsymbol{S}}=\mathscr{L}\left(2 \frac{\partial \bar{\psi}}{\partial \overline{\boldsymbol{C}}}\right)=\left.2 \frac{\partial \bar{\psi}}{\partial \overline{\boldsymbol{C}}}\right|_{\boldsymbol{I}}+\left.2 \frac{\partial^{2} \bar{\psi}}{\partial \overline{\boldsymbol{C}} \partial \overline{\boldsymbol{C}}}\right|_{\boldsymbol{I}}:\left.\frac{\partial \overline{\boldsymbol{C}}}{\partial \overline{\boldsymbol{F}}}\right|_{\boldsymbol{I}}: \overline{\operatorname{Grad}} \overline{\boldsymbol{u}},
$$

Following 43 and the definition of $\overline{\mathbb{I}}^{\text {sym }}$ we obtain

$$
\overline{\boldsymbol{\Sigma}}=\overline{\boldsymbol{\Pi}}_{0}+\overline{\mathbb{C}}: \overline{\mathbb{I}}^{\mathrm{sym}}: \overline{\operatorname{Grad}} \overline{\boldsymbol{u}} \quad \Rightarrow \quad \overline{\boldsymbol{\Sigma}}=\overline{\boldsymbol{\Pi}}_{0}+\overline{\mathbb{C}}: \overline{\boldsymbol{\epsilon}} .
$$

Similar to the finite strain (exact) theory, for the linearized theory we can derive the interface stress from the energy $\bar{\psi}_{\text {lin }}$ as

$$
\overline{\boldsymbol{\Sigma}}=\frac{\partial \bar{\psi}_{\text {lin }}}{\partial \overline{\boldsymbol{\epsilon}}} \quad \text { with } \quad \bar{\psi}_{\text {lin }}=\overline{\boldsymbol{\Pi}}_{0}: \overline{\boldsymbol{\epsilon}}+\frac{1}{2} \overline{\boldsymbol{\epsilon}}: \overline{\mathbb{C}}: \overline{\boldsymbol{\epsilon}} .
$$

From (53) and (44) we finally conclude that

$$
\overline{\boldsymbol{\Pi}}=\overline{\boldsymbol{\Sigma}}+\overline{\operatorname{Grad}} \overline{\boldsymbol{u}} \cdot \overline{\boldsymbol{\Pi}}_{0} .
$$

We observe that in this expression the term $\bar{\Sigma}$ is determined from the Helmholtz energy $\bar{\psi}_{\text {lin }}$ so that it gives the expression shown in (53). However, in relation to the previous discussion following (44) we again see clearly that the last term in 55 , i.e. $\overline{\text { Grad }} \overline{\boldsymbol{u}} \cdot \overline{\boldsymbol{\Pi}}_{0}$, is a result of the change of kinematics. 
Potential function for the interface stress $\bar{\Pi}$ differs from $\bar{\psi}_{\text {lin }}$. It is interesting to observe that $\bar{\Pi}$ given by (44) can be derived from the expression

$$
\overline{\boldsymbol{\Pi}}=\frac{\partial \bar{\phi}_{\text {lin }}}{\partial \overline{\operatorname{Grad}} \overline{\boldsymbol{u}}} \quad \text { with } \quad \bar{\phi}_{\text {lin }}=\bar{\psi}_{\text {lin }}+\frac{1}{2} \overline{\boldsymbol{\Pi}}_{0}:\left[[\overline{\operatorname{Grad}} \overline{\boldsymbol{u}}]^{\mathrm{t}} \cdot \overline{\operatorname{Grad}} \overline{\boldsymbol{u}}\right] .
$$

However, as emphasized above, the potential function $\bar{\phi}_{\text {lin }}$ is different from the Helmholtz energy $\bar{\psi}_{\text {lin }}$. Furthermore, potential $\bar{\phi}_{\text {lin }}$ is not invariant with respect to rigid body motions and more specifically to infinitesimal rotations. Clearly, the Helmholtz energy $\bar{\psi}_{\text {lin }}$ does fulfill the invariance properties with respect to rigid body motions.

Simplified versions of the interface potential $(\overline{56})$ are frequently used. The format of the energy (56) in various simplified forms is frequently employed in the literature. For instance, if we enforce the surface response to be isotropic and the initial stress to be $\overline{\boldsymbol{\Pi}}_{0}=\bar{\gamma} \overline{\boldsymbol{I}}$ with $\bar{\gamma}$ denoting the scalar-valued surface tension, the potential (56) shall be compared to Eq. (10) of [43]. The interface stress $\bar{\Pi}$ obviously includes the controversial non-symmetric gradient term.

Linearized stress measures coincide in the absence of the residual stress $\bar{\Pi}_{0}$. Let $\bar{\Pi}, \bar{\Sigma}$ and $\bar{\sigma}$ denote the linearized Piola, Piola-Kirchhoff and Cauchy stresses, respectively ${ }^{2}$ Via the linearization operator (34), one can show

$$
\overline{\boldsymbol{\Sigma}}=\overline{\boldsymbol{\Pi}}-\overline{\operatorname{Grad}} \overline{\boldsymbol{u}} \cdot \overline{\boldsymbol{\Pi}}_{0} \quad \text { and } \quad \overline{\boldsymbol{\sigma}}=\overline{\boldsymbol{\Pi}}-\overline{\boldsymbol{\Pi}}_{0} \cdot \overline{\operatorname{Grad}} \overline{\boldsymbol{u}}-\overline{\boldsymbol{\Pi}}_{0} \cdot\left[2 \overline{\mathbb{I}}^{\mathrm{vol}}-2 \overline{\mathbb{I}}^{\mathrm{sym}}\right]: \overline{\boldsymbol{\epsilon}},
$$

which clearly shows that various stress measures coincide when the interface residual stress $\bar{\Pi}_{0}$ vanishes.

Linearized isotropic interface energy reveals the connection to available studies. Almost all studies dealing with the interface or surface elasticity theory deal with the linearized isotropic case. Here, we show how our framework simplifies to this model. In order to derive the isotropic linear elasticity theory for interfaces, we start from the interface energy $\bar{\psi}$ being a function of invariants of $\overline{\boldsymbol{C}}$ as $\bar{\psi}=\bar{\psi}\left(\bar{I}_{1}, \bar{I}_{2}\right)$ with $\bar{I}_{1}=\overline{\boldsymbol{C}}: \overline{\boldsymbol{I}}$ and $\bar{I}_{2}=\overline{\text { Det }} \overline{\boldsymbol{C}}$. Next, we derive the constitutive tensor $\overline{\mathbb{C}}$ from this energy and insert it in Eq. 55 together with isotropic residual stress $\overline{\boldsymbol{\Pi}}_{0}=\bar{\gamma} \overline{\boldsymbol{I}}$ with $\bar{\gamma}$ denoting the scalar-valued surface tension. It is proven in Appendix $\mathrm{C}$ that the constitutive tensor $\overline{\mathbb{C}}$ reads

$$
\overline{\mathbb{C}}=2 \bar{\mu}_{\text {eff }} \overline{\mathbb{I}}^{\text {sym }}+2 \bar{\lambda}_{\text {eff }} \overline{\mathbb{I}}^{\mathrm{vol}} \quad \text { with } \quad \overline{\mathbb{I}}^{\mathrm{vol}}:=\frac{1}{2}[\overline{\boldsymbol{I}} \otimes \overline{\boldsymbol{I}}] \quad, \quad \overline{\mathbb{I}}^{\mathrm{sym}}:=\frac{1}{2}[\overline{\boldsymbol{I}} \bar{\otimes} \overline{\boldsymbol{I}}+\overline{\boldsymbol{I}} \otimes \overline{\boldsymbol{I}}] .
$$

Therefore, the interface stress $\bar{\Pi}$ simplifies to

$$
\overline{\boldsymbol{\Pi}}=\bar{\gamma} \overline{\boldsymbol{I}}+\left[2 \bar{\mu}_{\mathrm{eff}} \overline{\mathbb{T}}^{\mathrm{sym}}+2 \bar{\lambda}_{\mathrm{eff}} \overline{\mathbb{T}}^{\mathrm{vol}}\right]: \overline{\boldsymbol{\epsilon}}+\bar{\gamma} \overline{\mathrm{Grad}} \overline{\boldsymbol{u}},
$$

\footnotetext{
${ }^{2}$ The term Piola stress is used consistently instead of the commonly accepted first Piola-Kirchhoff stress. The term PiolaKirchhoff stress in this manuscript refers to the so-called second Piola-Kirchhoff stress which is symmetric and fully lies on the reference configuration. Cauchy stress is the classic symmetric stress in the current configuration.
} 
in which $\bar{\mu}_{\text {eff }}$ and $\bar{\lambda}_{\text {eff }}$ are the effective interface material parameters analogous to the Lamé parameters in the bulk. Inserting Eq. (57) into Eq. (59), clarifies why we introduce the effective interface material parameters to describe the linearized stress $\bar{\Pi}$ and how the material parameters are related to the surface tension $\bar{\gamma}$. In the case of isotropic interface behavior, $\overline{\boldsymbol{\Pi}}, \overline{\boldsymbol{\Sigma}}$ and $\overline{\boldsymbol{\sigma}}$ simplify to

$$
\begin{aligned}
& \overline{\boldsymbol{\Pi}}=\bar{\gamma} \overline{\boldsymbol{I}}+\left[2 \bar{\mu}_{\mathrm{eff}} \overline{\mathbb{I}}^{\mathrm{sym}}+2 \bar{\lambda}_{\mathrm{eff}} \overline{\mathbb{I}}^{\mathrm{vol}}\right]: \overline{\boldsymbol{\epsilon}}+\bar{\gamma} \overline{\mathrm{Grad}} \overline{\boldsymbol{u}}, \\
& \overline{\boldsymbol{\Sigma}}=\bar{\gamma} \overline{\boldsymbol{I}}+\left[2 \bar{\mu}_{\mathrm{eff}} \overline{\mathbb{I}}^{\mathrm{sym}}+2 \bar{\lambda}_{\mathrm{eff}} \overline{\mathbb{I}}^{\mathrm{vol}}\right]: \overline{\boldsymbol{\epsilon}}, \\
& \overline{\boldsymbol{\sigma}}=\bar{\gamma} \overline{\boldsymbol{I}}+\left[2\left[\bar{\mu}_{\mathrm{eff}}+\bar{\gamma}\right] \overline{\mathbb{I}}^{\mathrm{sym}}+2\left[\bar{\lambda}_{\mathrm{eff}}-\bar{\gamma}\right] \overline{\mathbb{I}}^{\mathrm{vol}}\right]: \overline{\boldsymbol{\epsilon}} .
\end{aligned}
$$

It is common practice to identify the material parameters based on the Cauchy stress as

$$
\overline{\boldsymbol{\sigma}}=\bar{\gamma} \overline{\boldsymbol{I}}+\left[2 \bar{\mu} \overline{\mathbb{I}}^{\mathrm{sym}}+2 \bar{\lambda} \overline{\mathbb{I}}^{\mathrm{vol}}\right]: \overline{\boldsymbol{\epsilon}}
$$

with $\bar{\mu}$ and $\bar{\lambda}$ being the interface Lamé parameters and, thus, the effective parameters can be identified as

$$
\bar{\mu}_{\text {eff }}=\bar{\mu}-\bar{\gamma} \quad, \quad \bar{\lambda}_{\text {eff }}=\bar{\lambda}+\bar{\gamma} .
$$

Inserting the effective parameters 62 into the linearized interface stress 59 furnishes

$$
\overline{\boldsymbol{\Pi}}=\bar{\gamma} \overline{\boldsymbol{I}}+\left[2[\bar{\mu}-\bar{\gamma}] \overline{\mathbb{I}}^{\mathrm{sym}}+2[\bar{\lambda}+\bar{\gamma}] \overline{\mathbb{I}}^{\mathrm{vol}}\right]: \overline{\boldsymbol{\epsilon}}+\bar{\gamma} \overline{\operatorname{Grad}} \overline{\boldsymbol{u}},
$$

or alternatively

$$
\overline{\boldsymbol{\Pi}}=\bar{\gamma} \overline{\boldsymbol{I}}+2[\bar{\mu}-\bar{\gamma}]: \overline{\boldsymbol{\epsilon}}+[\bar{\lambda}+\bar{\gamma}] \overline{\operatorname{Tr}} \overline{\boldsymbol{\epsilon}} \overline{\boldsymbol{I}}+\bar{\gamma} \overline{\operatorname{Grad}} \overline{\boldsymbol{u}} \quad \text { with } \quad \overline{\operatorname{Tr}} \overline{\boldsymbol{\epsilon}}=\overline{\boldsymbol{\epsilon}}: \overline{\boldsymbol{I}},
$$

which is precisely the interface stress as proposed by Gurtin and Murdoch [90. The mistake made by Gurtin and Murdoch in their widely-cited paper was to omit $\bar{\gamma}$ in the definition of the effective quantities. This error was rectified in an addendum [90] to their original work [6].

The non-symmetric term originates due to the fact that the stress-free and strain-free configurations do not coincide. In order to study this, we linearize the interface Piola stress $\overline{\boldsymbol{P}}$ at a stress-free configuration $\boldsymbol{F}^{*}$ as

$$
\overline{\boldsymbol{\Pi}}^{*}=\mathscr{L}^{*} \overline{\boldsymbol{P}}=\left.\overline{\boldsymbol{P}}\right|_{\overline{\boldsymbol{F}}^{*}}+\left.\frac{\partial \overline{\boldsymbol{P}}}{\partial \overline{\boldsymbol{F}}}\right|_{\overline{\boldsymbol{F}}^{*}}:\left[\overline{\boldsymbol{F}}-\overline{\boldsymbol{F}}^{*}\right]
$$

and since $\overline{\boldsymbol{P}}=\overline{\boldsymbol{F}} \cdot \overline{\boldsymbol{S}}$, we have

$$
\mathscr{L}^{*}(\overline{\boldsymbol{F}} \cdot \overline{\boldsymbol{S}})=\left.\mathscr{L}^{*} \overline{\boldsymbol{F}} \cdot \overline{\boldsymbol{S}}\right|_{\overline{\boldsymbol{F}}^{*}}+\left.\overline{\boldsymbol{F}}\right|_{\overline{\boldsymbol{F}}^{*}} \cdot \mathscr{L}^{*} \overline{\boldsymbol{S}}-\left.(\overline{\boldsymbol{F}} \cdot \overline{\boldsymbol{S}})\right|_{\overline{\boldsymbol{F}}^{*}}=\overline{\boldsymbol{F}} \cdot \overline{\boldsymbol{\Pi}}_{0}^{*}+\overline{\boldsymbol{F}}^{*} \cdot \mathscr{L}^{*} \overline{\boldsymbol{S}}-\overline{\boldsymbol{F}}^{*} \cdot \overline{\boldsymbol{\Pi}}_{0}^{*},
$$

in which the first and the last terms on the right-hand side vanish since the interface Piola stress $\bar{\Pi}_{0}^{*}$ at the configuration $\boldsymbol{F}^{*}$ is assumed to be zero and thus, we arrive at

$$
\overline{\boldsymbol{\Pi}}^{*}=\overline{\boldsymbol{F}}^{*} \cdot \mathscr{L}^{*} \overline{\boldsymbol{S}} .
$$


Equation 67] clearly projects the symmetric linearized stress $\mathscr{L}^{*} \overline{\boldsymbol{S}}$ onto the linear stress measure $\overline{\boldsymbol{\Pi}}^{*}$ whose divergence enters the balance of linear momentum on the interface. Note, that if $\overline{\boldsymbol{F}}^{*}$ is sufficiently close to the identity, the linearized interface stress $\overline{\boldsymbol{\Pi}}^{*}$ is sufficiently close to symmetry. It comes to be evident that in the limit of the stress-free configuration being strain-free, i.e. $\overline{\boldsymbol{F}}^{*}=\overline{\boldsymbol{I}}$, the linearized interface stress $\overline{\boldsymbol{\Pi}}^{*}$ becomes identically symmetric.

\section{Concluding remarks}

In this manuscript, we have presented a concise formulation of interface elasticity theory at finite deformations using two alternative notations. Various aspects and consequences of the interface elasticity theory are carefully examined and highlighted. Next, a consistent linearized interface elasticity theory is established. We propose an energetically consistent linear theory together with its appropriate stress measures. Our findings show that the controversial non-symmetric term in the linearized interface stress can play a crucial role in the balance of momentum at small strains.

In summary, this manuscript presents an attempt to shed light on interface elasticity theory in both finite and small deformations. The interface elasticity theory has received a particular attention in the past decade due its capabilities to capture the behavior of nano-materials and especially the size effect. We believe that our generic and consistent framework is broadly applicable to enhance our understanding of the behavior of continua with a large variety of applications.

\section{Appendix A. Differential geometry of interfaces}

It is enlightening to briefly review some basic terminologies and results on interfaces in the sense of twodimensional manifolds in three-dimensional space. For further details the reader is referred to [40, 42, 91, 92] among others. A two-dimensional (smooth) surface $\mathcal{I}$ in the three dimensional, embedding Euclidean space with coordinates $\overline{\mathbb{X}}$ is parameterized by two coordinates $\eta^{\alpha}$ with $\alpha=1,2$ as $\overline{\mathbb{X}}=\overline{\mathbb{X}}\left(\eta^{\alpha}\right)$. The corresponding tangent vectors $\bar{g}_{\alpha} \in T \mathcal{I}$ to the interface coordinate lines $\eta^{\alpha}$, i.e. the covariant (natural) interface basis vectors, are given by $\overline{\mathrm{g}}_{\alpha}=\partial_{\eta^{\alpha}} \overline{\mathrm{X}}$. The associated contravariant (dual) interface basis vectors $\overline{\mathrm{g}}^{\alpha}$ are defined by the Kronecker property $\delta_{\beta}^{\alpha}=\overline{\mathrm{g}}^{\alpha} \cdot \overline{\mathrm{g}}_{\beta}$ and are explicitly related to the covariant interface basis vectors $\overline{\mathrm{g}}_{\alpha}$ by the co- and contra-variant interface metric coefficients $\bar{g}_{\alpha \beta}$ (first fundamental form of the interface) and $\bar{g}^{\alpha \beta}$, respectively, as

$$
\begin{array}{lll}
\overline{\mathrm{g}}_{\alpha}=\bar{g}_{\alpha \beta} \overline{\mathrm{g}}^{\beta} & \text { with } & \bar{g}_{\alpha \beta}=\overline{\mathrm{g}}_{\alpha} \cdot \overline{\mathrm{g}}_{\beta}=\left[\bar{g}^{\alpha \beta}\right]^{-1}, \\
\overline{\mathrm{g}}^{\alpha}=\bar{g}^{\alpha \beta} \overline{\mathrm{g}}_{\beta} & \text { with } & \bar{g}^{\alpha \beta}=\overline{\mathrm{g}}^{\alpha} \cdot \overline{\mathrm{g}}^{\beta}=\left[\bar{g}_{\alpha \beta}\right]^{-1} .
\end{array}
$$

The contra- and covariant base vectors $\overline{\mathrm{g}}^{3}$ and $\overline{\mathrm{g}}_{3}$, normal to $T \mathcal{I}$, are defined by $\overline{\mathrm{g}}^{3}:=\overline{\mathrm{g}}_{1} \times \overline{\mathrm{g}}_{2}$ and $\overline{\mathrm{g}}_{3}:=\left[\bar{g}^{33}\right]^{-1} \overline{\mathrm{g}}^{3}$ so that $\overline{\mathrm{g}}^{3} \cdot \overline{\mathrm{g}}_{3}=1$. Thereby, the corresponding contra- and covariant metric coefficients, 


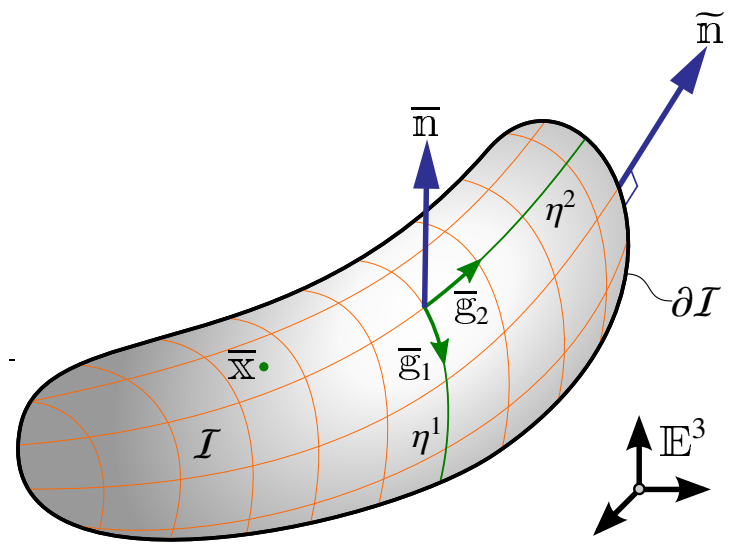

Figure A.3: The key differential geometry concepts of the interface as a two-dimensional manifolds in three-dimensional embedding Euclidean space $\mathbb{E}^{3}$. Coordinates $\overline{\mathbb{x}}$ can be parameterized by two coordinates $\eta^{1}$ and $\eta^{2}$ as $\overline{\mathbb{x}}=\overline{\mathbb{X}}\left(\eta^{1}, \eta^{2}\right)$. The covariance interface tangent vectors are denoted $\overline{\mathrm{g}}_{1}$ and $\overline{\mathrm{g}}_{2}$. The unit normal to the interface is denoted $\overline{\mathrm{n}}$. The outward unit normal to the boundary of the interface and tangential to the interface is denoted $\widetilde{\mathrm{n}}$.

respectively, $\left[\bar{g}^{33}\right]$ and $\left[\bar{g}_{33}\right]$ follow as

$$
\left[\bar{g}^{33}\right]=\left|\overline{\mathrm{g}}_{1} \times \overline{\mathrm{g}}_{2}\right|^{2}=\overline{\operatorname{det}}\left[\bar{g}_{\alpha \beta}\right]=\left[\overline{\operatorname{det}}\left[\bar{g}^{\alpha \beta}\right]\right]^{-1}=\left[\bar{g}_{33}\right]^{-1} .
$$

Accordingly, the interface area element $\mathrm{d} s$ and the interface normal $\overline{\mathrm{n}}$ are computed as

$$
\mathrm{d} s=\left|\overline{\mathrm{g}}_{1} \times \overline{\mathrm{g}}_{2}\right| \mathrm{d} \eta^{1} \mathrm{~d} \eta^{2}=\left[\bar{g}^{33}\right]^{1 / 2} \mathrm{~d} \eta^{1} \mathrm{~d} \eta^{2} \quad, \quad \overline{\mathrm{n}}=\left[\bar{g}_{33}\right]^{1 / 2} \overline{\mathrm{g}}^{3}=\left[\bar{g}^{33}\right]^{1 / 2} \overline{\mathrm{g}}_{3} .
$$

Moreover, with $\dot{i}$ denoting the ordinary mixed-variant unit tensor of the three-dimensional embedding Euclidian space, the mixed-variant interface unit tensor $\overline{\dot{I}}$ is defined as

$$
\overline{\mathrm{i}}:=\delta_{\beta}^{\alpha} \overline{\mathrm{g}}_{\alpha} \otimes \overline{\mathrm{g}}^{\beta}=\overline{\mathrm{g}}_{\alpha} \otimes \overline{\mathrm{g}}^{\alpha}=\dot{\mathrm{i}}-\overline{\mathrm{g}}_{3} \otimes \overline{\mathrm{g}}^{3}=\dot{\mathrm{i}}-\overline{\mathrm{m}} \otimes \overline{\mathrm{m}} .
$$

Clearly the mixed-variant interface unit tensor acts as an interface (idempotent) projection tensor. The interface gradient and interface divergence of a vector field $\{\bullet\}$ are defined by

$$
\overline{\operatorname{grad}}\{\bullet\}:=\partial_{\eta^{\alpha}}\{\bullet\} \otimes \overline{\mathrm{g}}^{\alpha} \quad, \quad \overline{\operatorname{div}}\{\bullet\}:=\partial_{\eta^{\alpha}}\{\bullet\} \cdot \overline{\mathrm{g}}^{\alpha} .
$$

As a consequence, observe that $\overline{\operatorname{grad}}\{\bullet\} \cdot \overline{\mathrm{m}}=\mathbf{0}$ holds by definition. For fields that are smooth in a neighbourhood of the interface, the interface gradient and interface divergence operators are alternatively defined as

$$
\overline{\operatorname{grad}}\{\bullet\}:=\operatorname{grad}\{\bullet\} \cdot \overline{\mathrm{I}} \quad, \quad \overline{\operatorname{div}}\{\bullet\}:=\overline{\operatorname{grad}}\{\bullet\}: \overline{\mathrm{I}}=\operatorname{grad}\{\bullet\}: \overline{\mathrm{i}} .
$$

Finally, the derivatives of the co- and contra-variant interface basis vectors read

$$
\partial_{\eta^{\beta}} \overline{\mathrm{g}}_{\alpha}=\Gamma_{\alpha \beta}^{\gamma} \overline{\mathrm{g}}_{\gamma}+\bar{k}_{\alpha \beta} \overline{\mathrm{M}} \quad, \quad \partial_{\eta^{\beta}} \overline{\mathrm{g}}^{\alpha}=-\Gamma_{\beta \gamma}^{\alpha} \overline{\mathrm{g}}^{\gamma}+\bar{k}_{\beta^{\alpha} \overline{\mathrm{M}}},
$$


where $\Gamma_{\alpha \beta}^{\gamma}=\partial_{\eta^{\beta}} \overline{\mathrm{g}}_{\alpha} \cdot \overline{\mathrm{g}}^{\gamma}$ denote the interface Christoffel symbols and $\bar{k}_{\alpha \beta}$ are the coefficients of the curvature tensor. The curvature tensor $\overline{\mathbb{k}}=\bar{k}_{\alpha \beta} \overline{\mathrm{g}}^{\alpha} \otimes \overline{\mathrm{g}}^{\beta}$ and twice the mean curvature ${ }^{3} \bar{k}=\bar{k}_{\alpha}^{\alpha}$ of the interface $\mathcal{I}$ are defined as the negative interface gradient and interface divergence of the interface normal $\overline{\mathrm{m}}$, respectively,

$$
\overline{\mathbb{k}}:=-\overline{\operatorname{grad}} \overline{\mathrm{m}}=-\partial_{\eta^{\beta}} \overline{\mathrm{m}} \otimes \overline{\mathrm{g}}^{\beta} \quad, \quad \bar{k}:=-\overline{\operatorname{div}} \overline{\overline{\mathrm{n}}}=-\partial_{\eta^{\beta}} \overline{\mathrm{m}} \cdot \overline{\mathrm{g}}^{\beta} .
$$

The covariant coefficients of the curvature tensor (second fundamental form of the interface) are computed by $\bar{k}_{\alpha \beta}=\overline{\mathrm{g}}_{\alpha} \cdot \overline{\mathrm{k}} \cdot \overline{\mathrm{g}}_{\beta}=-\overline{\mathrm{g}}_{\alpha} \cdot \partial_{\eta^{\beta}} \overline{\mathrm{n}}$.

For an arbitrary vector field $\overline{\mathbb{v}}$ tangential to the interface, i.e. $\overline{\mathbb{V}}=\overline{\mathbb{V}} \cdot \overline{\mathbb{i}}$, the interface divergence theorem reads

$$
\int_{\partial \mathcal{I}} \overline{\mathbb{V}} \cdot \widetilde{\mathrm{m}} \mathrm{d} l=\int_{\mathcal{I}} \overline{\operatorname{div}} \overline{\mathrm{V}} \mathrm{d} a \quad \quad \quad \text { with } \quad \overline{\mathbb{V}}=[\overline{\mathbb{V}}]^{\alpha} \overline{\mathrm{g}}_{\alpha} \quad, \quad \alpha \in\{1,2\},
$$

in which $\widetilde{\mathrm{m}}$ is the unit outward normal to the boundary of the interface but tangential to the interface. The interface divergence theorem A.9 is formally identical to the classic divergence theorem in the bulk since we a priori assumed that the vector field $\overline{\mathbb{v}}$ is tangential to the interface. Nevertheless, it is possible to establish another format of the interface divergence theorem for an arbitrary vector field $\overline{\mathbb{v}}$ not necessarily tangential to the interface. In doing so, we firstly decompose the vector $\overline{\mathbb{V}}$ to its tangential and orthogonal contributions according to

$$
\overline{\mathbb{V}}=\overline{\mathbb{V}} \cdot \overline{\mathrm{i}}+\overline{\mathbb{V}} \cdot[\overline{\mathrm{n}} \otimes \overline{\mathrm{n}}]
$$

and secondly, apply the interface divergence operator as

$$
\begin{aligned}
& \overline{\operatorname{div}} \overline{\mathbb{V}}=\overline{\operatorname{div}}(\overline{\mathbb{V}} \cdot \overline{\mathbb{i}})+\overline{\operatorname{div}}(\overline{\mathbb{V}} \cdot[\overline{\mathbf{n}} \otimes \overline{\mathrm{M}}]) \\
& =\overline{\operatorname{div}}(\overline{\mathbb{V}} \cdot \overline{\mathbb{i}})+\overline{\operatorname{grad}} \overline{\mathbb{v}}:[\overline{\mathrm{n}} \otimes \overline{\mathrm{m}}]+\overline{\mathbb{V}} \cdot \overline{\operatorname{grad}} \overline{\mathrm{n}} \cdot \overline{\mathrm{n}}+\overline{\operatorname{div}} \overline{\mathrm{n}} \overline{\mathbb{V}} \cdot \overline{\mathrm{m}},
\end{aligned}
$$

in which the second and the third terms on the right-hand side vanish due to the property $\overline{\operatorname{grad}}\{\bullet\} \cdot \overline{\mathrm{m}}=\mathbf{0}$ that holds by definition. Furthermore, $\overline{\operatorname{div}} \overline{\mathrm{n}}$ is minus twice the mean curvature and therefore,

$$
\overline{\operatorname{div}} \overline{\mathbb{V}}=\overline{\operatorname{div}}(\overline{\mathbb{V}} \cdot \overline{\mathrm{i}})-\bar{k} \overline{\mathbb{V}} \cdot \overline{\mathrm{M}} .
$$

Next, integrating the identity $\mathrm{A} .12$ over the interface furnishes

$$
\int_{\mathcal{I}} \overline{\operatorname{div}} \overline{\mathbb{V}} \mathrm{d} a=\int_{\mathcal{I}} \overline{\operatorname{div}}(\overline{\mathbb{V}} \cdot \overline{\mathrm{i}}) \mathrm{d} a-\int_{\mathcal{I}} \bar{k} \overline{\mathbb{V}} \cdot \overline{\mathrm{m}} \mathrm{d} a .
$$

\footnotetext{
3 There are various conventions to define the mean curvature in the literature. For instance, in 63 the term "mean curvature" refers to the sum of the principal curvatures or the trace of the curvature tensor. Here, we adopt another more intuitive definition of the mean curvature as the arithmetic mean of the principal curvatures and thus, $\bar{k}$ denotes twice the mean curvature.
} 
Since $\overline{\mathbb{V}} \cdot \overline{\mathbb{I}}$ is tangential to the interface, we can apply the interface divergence theorem A.9 on the first integral on the right-hand side and that renders

$$
\int_{\mathcal{I}} \overline{\operatorname{div}} \overline{\mathbb{V}} \mathrm{d} a=\int_{\partial \mathcal{I}}[\overline{\mathbb{V}} \cdot \overline{\mathrm{I}}] \cdot \widetilde{\mathrm{n}} \mathrm{d} l-\int_{\mathcal{I}} \bar{k} \overline{\mathbb{V}} \cdot \overline{\mathrm{m}} \mathrm{d} a .
$$

Note, without loss of generality, the relation $[\overline{\mathbb{V}} \cdot \overline{\mathrm{i}}] \cdot \widetilde{\mathbb{M}}=\overline{\mathbb{V}} \cdot \widetilde{\mathbb{M}}$ holds. Therefore, the interface divergence theorem for an arbitrary vector field $\overline{\mathbb{v}}$ not necessarily tangential to the interface reads

$$
\int_{\partial \mathcal{I}} \overline{\mathbb{V}} \cdot \widetilde{\mathrm{M}} \mathrm{d} l=\int_{\mathcal{I}} \overline{\operatorname{div}} \overline{\mathbb{V}} \mathrm{d} a+\int_{\mathcal{I}} \bar{k} \overline{\mathbb{V}} \cdot \overline{\mathrm{m}} \mathrm{d} a \quad \text { with } \quad \overline{\mathbb{V}}=[\overline{\mathbb{V}}]^{a} \overline{\mathrm{g}}_{a} \quad, \quad a \in\{1,2,3\} .
$$

In a near identical fashion, the interface divergence theorem for an arbitrary second-order tensor field $\overline{\mathbb{V}}$ not necessarily tangential to the interface reads

$$
\int_{\partial \mathcal{I}} \overline{\mathbb{V}} \cdot \widetilde{\mathrm{n}} \mathrm{d} l=\int_{\mathcal{I}} \overline{\operatorname{div}} \overline{\mathbb{V}} \mathrm{d} a+\int_{\mathcal{I}} \bar{k} \overline{\mathbb{V}} \cdot \overline{\mathrm{n}} \mathrm{d} a \quad \text { with } \quad \overline{\mathbb{V}}=[\overline{\mathbb{V}}]^{a b} \overline{\mathrm{g}}_{a} \otimes \overline{\mathrm{g}}_{b} \quad, \quad a, b \in\{1,2,3\} .
$$

From the format of Eq. A.16, it is clear that the integral containing the curvature vanishes if the secondorder tensor field $\overline{\mathbb{V}}$ is tangential to the interface only with respect to its second index. This particular family of second-order tensors play an important role in this contribution and are frequently referred to as superficial tensors according to $[6$. For instance, if $\overline{\mathbb{V}}$ is superficial, its projection onto the interface vanishes from right but not necessarily from left, i.e. $\overline{\mathbb{V}} \cdot \overline{\mathrm{i}}=\mathbf{0}$ but $\overline{\mathrm{i}} \cdot \overline{\mathbb{V}} \neq \mathbf{0}$, in general.

\section{Appendix B. Non-standard derivations on the interface}

The derivation procedures of various relations on the interface repeatedly boils down to carry out the derivation of $\partial \overline{\boldsymbol{n}} / \partial \overline{\boldsymbol{F}}$ which is elaborated in what follows. In order to derive $\partial \overline{\boldsymbol{n}} / \partial \overline{\boldsymbol{F}}$, first we note that $\partial \overline{\boldsymbol{n}} / \partial \overline{\boldsymbol{F}} \equiv \partial \overline{\boldsymbol{n}} / \partial \boldsymbol{F}$. More precisely

$$
\frac{\partial \overline{\boldsymbol{n}}}{\partial \boldsymbol{F}}=\frac{\partial \overline{\boldsymbol{n}}}{\partial \overline{\boldsymbol{F}}}: \frac{\partial \overline{\boldsymbol{F}}}{\partial \boldsymbol{F}}=\frac{\partial \overline{\boldsymbol{n}}}{\partial \overline{\boldsymbol{F}}}:[\boldsymbol{i} \bar{\otimes} \overline{\boldsymbol{I}}]=\frac{\partial \overline{\boldsymbol{n}}}{\partial \overline{\boldsymbol{F}}} \quad \text { with } \quad[\boldsymbol{i} \bar{\otimes} \overline{\boldsymbol{I}}]_{i j k l}=[\boldsymbol{i}]_{i k}[\overline{\boldsymbol{I}}]_{j l},
$$

or in index notation

$$
\left[\frac{\partial \overline{\boldsymbol{n}}}{\partial \boldsymbol{F}}\right]_{i j k}=\left[\frac{\partial \overline{\boldsymbol{n}}}{\partial \overline{\boldsymbol{F}}}\right]_{i r s}\left[\frac{\partial \overline{\boldsymbol{F}}}{\partial \boldsymbol{F}}\right]_{r s j k}=\left[\frac{\partial \overline{\boldsymbol{n}}}{\partial \overline{\boldsymbol{F}}}\right]_{i r s}\left[[\boldsymbol{i}]_{r j}[\overline{\boldsymbol{I}}]_{s k}\right]_{r s j k}=\left[\frac{\partial \overline{\boldsymbol{n}}}{\partial \overline{\boldsymbol{F}}}\right]_{i j k} .
$$

Considering an infinitesimal volume element and the definition of Jacobian, we have $\mathrm{d} v=J \mathrm{~d} V$ and the celebrated Nanson formula $\mathrm{d} \boldsymbol{a}=J \boldsymbol{F}^{-\mathrm{t}} \cdot \mathrm{d} \boldsymbol{A}$ or $\mathrm{d} \boldsymbol{a}=\operatorname{Cof} \boldsymbol{F} \cdot \mathrm{d} \boldsymbol{A}$ on the interface. Noting that $\mathrm{d} \boldsymbol{A}=\mathrm{d} A \overline{\boldsymbol{N}}$ and $\mathrm{d} \boldsymbol{a}=\mathrm{d} a \overline{\boldsymbol{n}}$ are the material and spatial area elements on the interface, respectively, we have

$$
\mathrm{d} a \overline{\boldsymbol{n}}=J \boldsymbol{F}^{-\mathrm{t}} \cdot[\mathrm{d} A \overline{\boldsymbol{N}}] \quad \Rightarrow \quad \overline{\boldsymbol{n}}=J \boldsymbol{F}^{-\mathrm{t}} \cdot \overline{\boldsymbol{N}} \frac{\mathrm{d} A}{\mathrm{~d} a}=\frac{J}{\bar{J}} \boldsymbol{F}^{-\mathrm{t}} \cdot \overline{\boldsymbol{N}} \quad \text { or } \quad \overline{\boldsymbol{n}}=\frac{\boldsymbol{F}^{-\mathrm{t}} \cdot \overline{\boldsymbol{N}}}{\left|\boldsymbol{F}^{-\mathrm{t}} \cdot \overline{\boldsymbol{N}}\right|} .
$$

Recalling that $\bar{J}=\mathrm{d} a / \mathrm{d} A$ denotes the interface Jacobian. We proceed using the identities

$$
\frac{\partial}{\partial \boldsymbol{F}}\left(\frac{\boldsymbol{u}}{|\boldsymbol{u}|}\right)=\frac{1}{|\boldsymbol{u}|}\left[\boldsymbol{i}-\frac{\boldsymbol{u}}{|\boldsymbol{u}|} \otimes \frac{\boldsymbol{u}}{|\boldsymbol{u}|}\right] \cdot \frac{\partial \boldsymbol{u}}{\partial \boldsymbol{F}} \quad, \quad \frac{\partial|\boldsymbol{u}|}{\partial \boldsymbol{F}}=\frac{\boldsymbol{u}}{|\boldsymbol{u}|} \cdot \frac{\partial \boldsymbol{u}}{\partial \boldsymbol{F}} \quad \forall \boldsymbol{u}: \text { arbitrary vector }
$$


and therefore

$$
\frac{\partial \overline{\boldsymbol{n}}}{\partial \boldsymbol{F}}=\frac{\partial}{\partial \boldsymbol{F}}\left(\frac{\boldsymbol{F}^{-\mathrm{t}} \cdot \overline{\boldsymbol{N}}}{\left|\boldsymbol{F}^{-\mathrm{t}} \cdot \overline{\boldsymbol{N}}\right|}\right)=\frac{1}{\left|\boldsymbol{F}^{-\mathrm{t}} \cdot \overline{\boldsymbol{N}}\right|}[\boldsymbol{i}-\overline{\boldsymbol{n}} \otimes \overline{\boldsymbol{n}}] \cdot \frac{\partial \boldsymbol{F}^{-\mathrm{t}} \cdot \overline{\boldsymbol{N}}}{\partial \boldsymbol{F}},
$$

using the definition of the interface identity in the spatial configuration $\overline{\boldsymbol{i}}=\boldsymbol{i}-\overline{\boldsymbol{n}} \otimes \overline{\boldsymbol{n}}$ together with the identities $\partial \boldsymbol{F}^{-\mathrm{t}} / \partial \boldsymbol{F}=-\boldsymbol{F}^{-\mathrm{t}} \underline{\otimes} \boldsymbol{F}^{-1}$ or $\partial \boldsymbol{F}^{-1} / \partial \boldsymbol{F}=-\boldsymbol{F}^{-1} \bar{\otimes} \boldsymbol{F}^{-\mathrm{t}}$ with $\left[\boldsymbol{F}^{-\mathrm{t}} \underline{\otimes} \boldsymbol{F}^{-1}\right]_{i j k l}=\left[\boldsymbol{F}^{-\mathrm{t}}\right]_{i l}\left[\boldsymbol{F}^{-1}\right]_{j k}$,

$$
=-\frac{1}{\left|\boldsymbol{F}^{-\mathrm{t}} \cdot \overline{\boldsymbol{N}}\right|} \overline{\boldsymbol{i}} \cdot\left[\boldsymbol{F}^{-\mathrm{t}} \bar{\otimes}\left[\boldsymbol{F}^{-\mathrm{t}} \cdot \overline{\boldsymbol{N}}\right]\right]=-\overline{\boldsymbol{i}} \cdot\left[\boldsymbol{F}^{-\mathrm{t}} \bar{\otimes} \overline{\boldsymbol{n}}\right]=-\underbrace{\left[\overline{\boldsymbol{i}} \cdot \boldsymbol{F}^{-\mathrm{t}}\right]}_{\overline{\boldsymbol{F}^{-\mathrm{t}}}} \bar{\otimes} \overline{\boldsymbol{n}}=-\overline{\boldsymbol{F}}^{-\mathrm{t}} \bar{\otimes} \overline{\boldsymbol{n}},
$$

in which the relation $\overline{\boldsymbol{F}}^{\text {-t }}=\overline{\boldsymbol{i}} \cdot \boldsymbol{F}^{-\mathrm{t}}$ follows as the transpose of $\overline{\boldsymbol{F}}^{-1}=\boldsymbol{F}^{-1} \cdot \overline{\boldsymbol{i}}$. Therefore

$$
\frac{\partial \overline{\boldsymbol{n}}}{\partial \overline{\boldsymbol{F}}} \equiv \frac{\partial \overline{\boldsymbol{n}}}{\partial \boldsymbol{F}}=-\overline{\boldsymbol{n}} \bar{\otimes} \overline{\boldsymbol{F}}^{-\mathrm{t}} \quad \Rightarrow \quad\left[\frac{\partial \overline{\boldsymbol{n}}}{\partial \overline{\boldsymbol{F}}}\right]_{i j k}=-[\overline{\boldsymbol{n}}]_{j}\left[\overline{\boldsymbol{F}}^{-\mathrm{t}}\right]_{i k}=-[\overline{\boldsymbol{n}}]_{j}\left[\overline{\boldsymbol{F}}^{-1}\right]_{k i} .
$$

An important consequence of this relation is the derivative of the spatial interface identity with respect to the deformation gradient as

$$
\frac{\partial \overline{\boldsymbol{i}}}{\partial \overline{\boldsymbol{F}}}=\frac{\partial \overline{\boldsymbol{i}}}{\partial \boldsymbol{F}}=\frac{\partial(\boldsymbol{i}-\overline{\boldsymbol{n}} \otimes \overline{\boldsymbol{n}})}{\partial \overline{\boldsymbol{F}}}=-\frac{\partial(\overline{\boldsymbol{n}} \otimes \overline{\boldsymbol{n}})}{\partial \overline{\boldsymbol{F}}}=-[\overline{\boldsymbol{n}} \otimes \overline{\boldsymbol{n}}] \bar{\otimes} \overline{\boldsymbol{F}}^{-\mathrm{t}}-\overline{\boldsymbol{F}}^{\mathrm{t}} \underline{\otimes}[\overline{\boldsymbol{n}} \otimes \overline{\boldsymbol{n}}],
$$

or in index notation

$$
\left[\frac{\partial \overline{\boldsymbol{i}}}{\partial \overline{\boldsymbol{F}}}\right]_{i j k l}=\left[\frac{\partial \overline{\boldsymbol{i}}}{\partial \boldsymbol{F}}\right]_{i j k l}=-[\overline{\boldsymbol{n}}]_{i}[\overline{\boldsymbol{n}}]_{k}\left[\overline{\boldsymbol{F}}^{-\mathrm{t}}\right]_{j l}-\left[\overline{\boldsymbol{F}}^{-\mathrm{t}}\right]_{i l}[\overline{\boldsymbol{n}}]_{k}[\overline{\boldsymbol{n}}]_{j} .
$$

\section{Appendix C. Derivation of linearized isotropic constitutive tensor}

Here, we detail on the derivations of the interface linearized isotropic constitutive tensor $\overline{\mathbb{C}}$ from its energy $\bar{\psi}=\bar{\psi}\left(\bar{I}_{1}, \bar{I}_{2}\right)$ with $\bar{I}_{1}$ and $\bar{I}_{2}$ being the invariants $\overline{\boldsymbol{C}}$. To do so, recall that

$$
\bar{I}_{1}=\overline{\boldsymbol{C}}: \overline{\boldsymbol{I}} \quad \Rightarrow \quad \frac{\partial \bar{I}_{1}}{\partial \overline{\boldsymbol{C}}}=\overline{\boldsymbol{I}} \quad \text { and } \quad \bar{I}_{2}=\overline{\operatorname{Det}} \overline{\boldsymbol{C}} \quad \Rightarrow \quad \frac{\partial \bar{I}_{2}}{\partial \overline{\boldsymbol{C}}}=\bar{I}_{2} \overline{\boldsymbol{C}}^{-1} .
$$

Therefore,

$$
\begin{aligned}
& \overline{\mathbb{C}}=\left.4 \frac{\partial^{2} \bar{\psi}}{\partial \overline{\boldsymbol{C}} \partial \overline{\boldsymbol{C}}}\right|_{\boldsymbol{I}}=\left.4 \frac{\partial}{\partial \overline{\boldsymbol{C}}}\left(\frac{\partial \bar{\psi}}{\partial \bar{I}_{1}} \frac{\partial \bar{I}_{1}}{\partial \overline{\boldsymbol{C}}}+\frac{\partial \bar{\psi}}{\partial \bar{I}_{2}} \frac{\partial \bar{I}_{2}}{\partial \overline{\boldsymbol{C}}}\right)\right|_{\boldsymbol{I}}=\left.4 \frac{\partial}{\partial \overline{\boldsymbol{C}}}\left(\frac{\partial \bar{\psi}}{\partial \bar{I}_{1}} \overline{\boldsymbol{I}}+\frac{\partial \bar{\psi}}{\partial \bar{I}_{2}} \bar{I}_{2} \overline{\boldsymbol{C}}^{-1}\right)\right|_{\boldsymbol{I}} \\
& =\left.4 \overline{\boldsymbol{I}} \otimes \frac{\partial}{\partial \overline{\boldsymbol{C}}}\left(\frac{\partial \bar{\psi}}{\partial \bar{I}_{1}}\right)\right|_{\boldsymbol{I}}+\left.4 \overline{\boldsymbol{C}}^{-1} \otimes \frac{\partial}{\partial \overline{\boldsymbol{C}}}\left(\frac{\partial \bar{\psi}}{\partial \bar{I}_{2}} \bar{I}_{2}\right)\right|_{\boldsymbol{I}}+\left.4 \frac{\partial \bar{\psi}}{\partial \bar{I}_{2}} \bar{I}_{2} \frac{\partial \overline{\boldsymbol{C}}^{-1}}{\partial \overline{\boldsymbol{C}}}\right|_{\boldsymbol{I}} \\
& =\left.4 \overline{\boldsymbol{I}} \otimes \frac{\partial \bar{I}_{1}}{\partial \overline{\boldsymbol{C}}} \frac{\partial^{2} \bar{\psi}}{\partial \bar{I}_{1}^{2}}\right|_{\boldsymbol{I}}+\left.4 \overline{\boldsymbol{I}} \otimes \frac{\partial \bar{I}_{2}}{\partial \overline{\boldsymbol{C}}} \frac{\partial^{2} \bar{\psi}^{2}}{\partial \bar{I}_{2} \partial \bar{I}_{1}}\right|_{\boldsymbol{I}}+\left.4 \overline{\boldsymbol{C}}^{-1} \otimes \frac{\partial \bar{I}_{1}}{\partial \overline{\boldsymbol{C}}} \frac{\partial}{\partial \bar{I}_{1}}\left(\frac{\partial \bar{\psi}}{\partial \bar{I}_{2}} \bar{I}_{2}\right)\right|_{\boldsymbol{I}} \\
& +\left.4 \overline{\boldsymbol{C}}^{-1} \otimes \frac{\partial \bar{I}_{2}}{\partial \overline{\boldsymbol{C}}} \frac{\partial}{\partial \bar{I}_{2}}\left(\frac{\partial \bar{\psi}}{\partial \bar{I}_{2}} \bar{I}_{2}\right)\right|_{\boldsymbol{I}}-\left.4 \frac{\partial \bar{\psi}}{\partial \bar{I}_{2}}\right|_{\boldsymbol{I}} \overline{\mathbb{I}}^{\text {sym }} \\
& =\left.4 \overline{\boldsymbol{I}} \otimes \overline{\boldsymbol{I}} \frac{\partial^{2} \bar{\psi}}{\partial \bar{I}_{1}^{2}}\right|_{\boldsymbol{I}}+\left.8 \overline{\boldsymbol{I}} \otimes \overline{\boldsymbol{I}} \frac{\partial^{2} \bar{\psi}}{\partial \bar{I}_{1} \partial \bar{I}_{2}}\right|_{\boldsymbol{I}}+\left.4 \overline{\boldsymbol{I}} \otimes \overline{\boldsymbol{I}} \frac{\partial^{2} \bar{\psi}}{\partial \bar{I}_{2}^{2}}\right|_{\boldsymbol{I}}+\left.4 \overline{\boldsymbol{I}} \otimes \overline{\boldsymbol{I}} \frac{\partial \bar{\psi}}{\partial \bar{I}_{2}}\right|_{\boldsymbol{I}}-\left.4 \frac{\partial \bar{\psi}}{\partial \bar{I}_{2}}\right|_{\boldsymbol{I}} \overline{\boldsymbol{I}}^{\mathrm{sym}} \\
& =2 \underbrace{\left[\left.4 \frac{\partial^{2} \bar{\psi}}{\partial \bar{I}_{1}^{2}}\right|_{\boldsymbol{I}}+\left.8 \frac{\partial^{2} \bar{\psi}}{\partial \bar{I}_{1} \partial \bar{I}_{2}}\right|_{\boldsymbol{I}}+\left.4 \frac{\partial^{2} \bar{\psi}}{\partial \bar{I}_{2}^{2}}\right|_{\boldsymbol{I}}+\left.4 \frac{\partial \bar{\psi}}{\partial \bar{I}_{2}}\right|_{\boldsymbol{I}}\right]}_{\bar{\lambda}_{\text {eff }}} \overline{\mathbb{I}}^{\mathrm{vol}}+2 \underbrace{\left[-\left.2 \frac{\partial \bar{\psi}}{\partial \bar{I}_{2}}\right|_{\boldsymbol{I}}\right]}_{\bar{\mu}_{\mathrm{eff}}} \overline{\mathbb{I}}^{\mathrm{sym}} \\
& =2 \bar{\lambda}_{\mathrm{eff}} \overline{\mathbb{I}}^{\mathrm{vol}}+2 \bar{\mu}_{\mathrm{eff}} \overline{\bar{I}}^{\mathrm{sym}} \quad \text { with } \quad \overline{\mathbb{I}}^{\mathrm{vol}}:=\frac{1}{2}[\overline{\boldsymbol{I}} \otimes \overline{\boldsymbol{I}}] \quad \text { and } \quad \overline{\mathbb{I}}^{\mathrm{sym}}:=\frac{1}{2}[\overline{\boldsymbol{I}} \bar{\otimes} \overline{\boldsymbol{I}}+\overline{\boldsymbol{I}} \underline{\otimes} \overline{\boldsymbol{I}}] .
\end{aligned}
$$




\section{References}

[1] Z. Hashin, S. Shtrikman, A variational approach to the theory of the elastic behaviour of multiphase materials, Journal of the Mechanics and Physics of Solids 11 (2) (1963) 127-140.

[2] Z. Hashin, Thin interphase/imperfect interface in elasticity with application to coated fiber composites, Journal of the Mechanics and Physics of Solids 50 (12) (2002) 2509-2537.

[3] Y. Benveniste, O. Berdichevsky, On two models of arbitrarily curved three-dimensional thin interphases in elasticity, International Journal of Solids and Structures 47 (14-15) (2010) 1899-1915.

[4] S. T. Gu, Q. C. He, Interfacial discontinuity relations for coupled multifield phenomena and their application to the modeling of thin interphases as imperfect interfaces, Journal of the Mechanics and Physics of Solids 59 (7) (2011) 14131426.

[5] J. Mosler, O. Shchyglo, H. Montazer Hojjat, A novel homogenization method for phase field approaches based on partial rank-one relaxation, Journal of the Mechanics and Physics of Solids 68 (1) (2014) 251-266.

[6] M. E. Gurtin, A. I. Murdoch, A continuum theory of elastic material surfaces, Archive for Rational Mechanics and Analysis 57 (4) (1975) 291-323.

[7] A. I. Murdoch, A thermodynamical theory of elastic material interfaces, Quarterly Journal of Mechanics and Applied Mathematics 29 (3) (1976) 245-275.

[8] S. Iijima, Helical microtubules of graphitic carbon, Nature 354 (6348) (1991) 56-58.

[9] E. W. Wong, Nanobeam Mechanics: Elasticity, Strength, and Toughness of Nanorods and Nanotubes, Science 277 (5334) (1997) 1971-1975.

[10] A. M. Morales, A Laser Ablation Method for the Synthesis of Crystalline Semiconductor Nanowires, Science 279 (5348) (1998) 208-211.

[11] K. S. Kumar, H. Van Swygenhoven, S. Suresh, Mechanical behavior of nanocrystalline metals and alloys, Acta Materialia 51 (19) (2003) 5743-5774.

[12] X. Y. Kong, Z. L. Wang, Spontaneous Polarization-Induced Nanohelixes, Nanosprings, and Nanorings of Piezoelectric Nanobelts, Nano Letters 3 (12) (2003) 1625-1631.

[13] V. Ramaswamy, W. D. Nix, B. M. Clemens, Coherency and surface stress effects in metal multilayers, Scripta Materialia 50 (6) (2004) 711-715.

[14] R. E. Miller, V. B. Shenoy, Size-dependent elastic properties of nanosized structural elements, Nanotechnology 11 (3) (2000) 139-147.

[15] P. Sharma, S. Ganti, N. Bhate, Effect of surfaces on the size-dependent elastic state of nano-inhomogeneities, Applied Physics Letters 82 (4) (2003) 535-537.

[16] H. S. Park, P. Klein, G. Wagner, A surface Cauchy-Born model for nanoscale materials, International Journal for Numerical Methods in Engineering 68 (10) (2006) 1072-1095.

[17] H. L. Duan, J. Wang, B. L. Karihaloo, Theory of Elasticity at the Nanoscale, Advances in Applied Mechanics 42 (2009) $1-68$.

[18] J. Yvonnet, A. Mitrushchenkov, G. Chambaud, Q. C. He, S. T. Gu, Characterization of surface and nonlinear elasticity in wurtzite ZnO nanowires, Journal of Applied Physics 111 (2012) 124305.

[19] D. Davydov, A. Javili, P. Steinmann, On molecular statics and surface-enhanced continuum modeling of nano-structures, Computational Materials Science 69 (2013) 510-519.

[20] S. Rezazadeh Kalehbasti, M. Y. Gutkin, H. M. Shodja, Wedge disclinations in the shell of a core-shell nanowire within the surface/interface elasticity, Mechanics of Materials 68 (2014) 45-63.

[21] G. Chatzigeorgiou, A. Javili, P. Steinmann, Surface electrostatics : theory and computations, Proceedings of the Royal Society A: Mathematical, Physical and Engineering Sciences 470 (2014) 20130628. 
[22] L. Hu, L. Liu, From atomistics to continuum: Effects of a free surface and determination of surface elasticity properties, Mechanics of Materials 90 (2015) 202-211.

[23] A. Javili, G. Chatzigeorgiou, A. T. McBride, P. Steinmann, C. Linder, Computational homogenization of nano-materials accounting for size effects via surface elasticity, GAMM Mitteilungen 38 (2) (2015) 285-312.

[24] N. Daher, G. A. Maugin, The method of virtual power in continuum mechanics application to media presenting singular surfaces and interfaces, Acta Mechanica 60 (3-4) (1986) 217-240.

[25] R. Shuttleworth, The Surface Tension of Solids, Proceedings of the Physics Society A 63 (1950) 444-457.

[26] E. Orowan, Surface Energy and Surface Tension in Solids and Liquids, Proceedings of the Royal Society A: Mathematical, Physical and Engineering Sciences 316 (1970) 473-491.

[27] T. Chen, M. S. Chiu, C. N. Weng, Derivation of the generalized Young-Laplace equation of curved interfaces in nanoscaled solids, Journal of Applied Physics 100 (7) (2006) 074308.

[28] G. P. Moeckel, Thermodynamics of an interface, Archive for Rational Mechanics and Analysis 57 (3) (1975) 255-280.

[29] F. Dell'Isola, A. Romano, On the derivation of thermomechanical balance equations for continuous systems with a nonmaterial interface, International Journal of Engineering Science 25 (11-12) (1987) 1459-1468.

[30] D. J. Steigmann, R. W. Ogden, Elastic surface-substrate interactions, Proceedings of the Royal Society A: Mathematical, Physical and Engineering Sciences 455 (1982) (1999) 437-474.

[31] E. Fried, R. E. Todres, Mind the gap: The shape of the free surface of a rubber-like material in proximity to a rigid contactor, Journal of Elasticity 80 (1-3) (2005) 97-151.

[32] E. Fried, M. E. Gurtin, Thermomechanics of the interface between a body and its environment, Continuum Mechanics and Thermodynamics 19 (5) (2007) 253-271.

[33] M. Silhavy, Equilibrium of phases with interfacial energy: A variational approach, Journal of Elasticity 105 (1-2) (2011) 271-303.

[34] P. Chhapadia, P. Mohammadi, P. Sharma, Curvature-dependent surface energy and implications for nanostructures, Journal of the Mechanics and Physics of Solids 59 (10) (2011) 2103-2115.

[35] A. T. McBride, A. Javili, P. Steinmann, S. Bargmann, Geometrically nonlinear continuum thermomechanics with surface energies coupled to diffusion, Journal of the Mechanics and Physics of Solids 59 (10) (2011) 2116-2133.

[36] A. Javili, A. Mcbride, P. Steinmann, Thermomechanics of Solids With Lower-Dimensional Energetics: On the Importance of Surface, Interface, and Curve Structures at the Nanoscale. A Unifying Review, Applied Mechanics Reviews 65 (1) (2013) 010802 .

[37] R. Dingreville, A. Hallil, S. Berbenni, From coherent to incoherent mismatched interfaces: A generalized continuum formulation of surface stresses, Journal of the Mechanics and Physics of Solids 72 (1) (2014) 40-60.

[38] X. Gao, Z. Huang, J. Qu, D. Fang, A curvature-dependent interfacial energy-based interface stress theory and its applications to nano-structured materials: (I) General theory, Journal of the Mechanics and Physics of Solids 66 (1) (2014) $59-77$.

[39] M. Serpilli, Asymptotic interface models in magneto-electro-thermo-elastic composites, Meccanicrdoi:10.1007/ s11012-016-0481-4

[40] P. G. Ciarlet, An Introduction to Differential Geometry with Applications to Elasticity, Springer, 2005.

[41] A. Javili, A. McBride, P. Steinmann, B. D. Reddy, A unified computational framework for bulk and surface elasticity theory: a curvilinear-coordinate-based finite element methodology, Computational Mechanics 54 (2014) 745-762.

[42] P. Steinmann, Geometrical Foundations of Continuum Mechanics: An Application to First- and Second-Order Elasticity and Elasto-Plasticity, Springer, 2015.

[43] C. Q. Ru, Simple geometrical explanation of Gurtin-Murdoch model of surface elasticity with clarification of its related versions, Science China: Physics, Mechanics and Astronomy 53 (3) (2010) 536-544. 
[44] G. I. Barenblatt, The formation of equilibrium cracks during brittle fracture. General ideas and hypotheses. Axiallysymmetric cracks, Journal of Applied Mathematics and Mechanics 23 (3) (1959) 622-636.

[45] G. I. Barenblatt, The Mathematical Theory of Equilibrium Cracks in Brittle Fracture, Advances in Applied Mechanics 7 (1962) 55-129.

[46] D. Dugdale, Yielding of steel sheets containing slits, Journal of the Mechanics and Physics of Solids 8 (2) (1960) 100-104.

[47] A. Hillerborg, M. Modéer, P.-E. Petersson, Analysis of crack formation and crack growth in concrete by means of fracture mechanics and finite elements, Cement and Concrete Research 6 (1976) 773-781.

[48] A. Needleman, A Continuum Model for Void Nucleation by Inclusion Debonding, Journal of Applied Mechanics 54 (1987) $525-531$

[49] X.-P. Xu, A. Needleman, Numerical simulations of fast crack growth in brittle solids, Journal of the Mechanics and Physics of Solids 42 (9) (1994) 1397-1434.

[50] M. Ortiz, A. Pandolfi, Finite-deformation irreversible cohesive elements for three-dimensional crack-propagation analysis, International Journal for Numerical Methods in Engineering 44 (1999) 1267-1282.

[51] G. Alfano, M. A. Crisfield, Finite element interface models for the delamination analysis of laminated composites: mechanical and computational issues, Int. J. Numer. Meth. Eng. 50 (2001) 1701-1736.

[52] T. C. Gasser, G. A. Holzapfel, Geometrically non-linear and consistently linearized embedded strong discontinuity models for 3D problems with an application to the dissection analysis of soft biological tissues, Computer Methods in Applied Mechanics and Engineering 192 (47-48) (2003) 5059-5098.

[53] M. J. van den Bosch, P. J. G. Schreurs, M. G. D. Geers, An improved description of the exponential Xu and Needleman cohesive zone law for mixed-mode decohesion, Engineering Fracture Mechanics 73 (9) (2006) 1220-1234.

[54] M. Fagerström, R. Larsson, Theory and numerics for finite deformation fracture modelling using strong discontinuities, International Journal for Numerical Methods in Engineering 66 (6) (2006) 911-948.

[55] K. Park, G. H. Paulino, J. R. Roesler, A unified potential-based cohesive model of mixed-mode fracture, Journal of the Mechanics and Physics of Solids 57 (6) (2009) 891-908.

[56] J. Mosler, I. Scheider, A thermodynamically and variationally consistent class of damage-type cohesive models, Journal of the Mechanics and Physics of Solids 59 (8) (2011) 1647-1668.

[57] A. McBride, J. Mergheim, A. Javili, P. Steinmann, S. Bargmann, Micro-to-macro transitions for heterogeneous material layers accounting for in-plane stretch, Journal of the Mechanics and Physics of Solids 60 (6) (2012) 1221-1239.

[58] K. Park, G. H. Paulino, Cohesive Zone Models : A Critical Review of Traction-Separation Relationships Across Fracture Surfaces, Applied Mechanics Reviews 64 (6) (2013) 060802.

[59] R. Dimitri, M. Trullo, L. De Lorenzis, G. Zavarise, Coupled cohesive zone models for mixed-mode fracture: A comparative study, Engineering Fracture Mechanics 148 (2015) 145-179.

[60] N. S. Ottosen, M. Ristinmaa, J. Mosler, Fundamental physical principles and cohesive zone models at finite displacements - Limitations and possibilities, International Journal of Solids and Structures 53 (2015) 70-79.

[61] A. Esmaeili, A. Javili, P. Steinmann, A thermo-mechanical cohesive zone model accounting for mechanically energetic Kapitza interfaces, International Journal of Solids and Structures 92-93 (2016) 29-44.

[62] E. Lejeune, A. Javili, C. Linder, Understanding geometric instabilities in thin films via a multi-layer model, Soft Matter $12(2016) 806$.

[63] J. E. Marsden, T. J. R. Hughes, Mathematical Foundations of Elasticity, Dover, 1994.

[64] G. A. Holzapfel, Nonlinear Solid Mechanics: A Continuum Approach for Engineering, John Wiley \& Sons, 2000.

[65] M. E. Gurtin, E. Fried, L. Anand, The Mechanics and Thermodynamics of Continua, Cambridge University Press, 2009.

[66] A. Javili, F. Dell'Isola, P. Steinmann, Geometrically nonlinear higher-gradient elasticity with energetic boundaries, Journal of the Mechanics and Physics of Solids 61 (12) (2013) 2381-2401. 
[67] N. M. Cordero, S. Forest, E. P. Busso, Second strain gradient elasticity of nano-objects, Journal of the Mechanics and Physics of Solids 97 (2016) 92-124.

[68] N. S. Ottosen, M. Ristinmaa, J. Mosler, Framework for non-coherent interface models at finite displacement jumps and finite strains, Journal of the Mechanics and Physics of Solids 90 (2016) 124-141.

[69] S. Angenent, M. E. Gurtin, Multiphase thermomechanics with interfacial structure 2. Evolution of an isothermal interface, Archive for Rational Mechanics and Analysis 108 (3) (1989) 323-391.

[70] M. E. Gurtin, A. Struthers, Multiphase thermomechanics with interfacial structure - 3. Evolving phase boundaries in the presence of bulk deformation, Archive for Rational Mechanics and Analysis 112 (2) (1990) 97-160.

[71] P. Steinmann, On boundary potential energies in deformational and configurational mechanics, Journal of the Mechanics and Physics of Solids 56 (3) (2008) 772-800.

[72] M. E. Gurtin, Configurational Forces as Basic Concepts of Continuum Physics, 2000.

[73] N. K. Simha, K. Bhattacharya, Kinetics of phase boundaries with edges and junctions, J .Mech. Phys. Solids 46 (12) (1998) 2323-2359.

[74] N. K. Simha, K. Bhattacharya, Kinetics of phase boundaries with edges and junctions in a three-dimensional multi-phase body, Journal of the Mechanics and Physics of Solids 48 (12) (2000) 2619-2641.

[75] E. Fried, M. E. Gurtin, The role of the configurational force balance in the nonequilibrium epitaxy of films, Journal of the Mechanics and Physics of Solids 51 (3) (2003) 487-517.

[76] T. Alts, K. Hutter, Continuum Description of the Dynamics and Thermodynamics of Phase Boundaries Between Ice and Water, Part I, Journal of Non-Equilibrium Thermodynamics 13 (3) (1988) 221-257.

[77] E. Fried, M. E. Gurtin, A unified treatment of evolving interfaces accounting for small deformations and atomic transport with emphasis on grain-boundaries and epitaxy, Advances in applied mechanics 40 (2004) 1-177.

[78] M. E. Gurtin, A general theory of curved deformable interfaces in solids at equilibrium, Philosophical Magazine A 78 (5) (1998) 1093-1109. doi:10.1080/014186198253138

[79] D. J. Steigmann, Thin-plate theory for large elastic deformations, International Journal of Non-Linear Mechanics 42 (2) (2007) 233-240.

[80] Y. Z. Povstenko, Theoretical investigation of phenomena caused by heterogenous surface tension in solids, Journal of the Mechanics and Physics of Solids 41 (9) (1993) 1499-1514.

[81] E. M. Gutman, On the thermodynamic definition of surface stress, Journal of Physics: Condensed Matter 7 (48) (1995) L663-L667.

[82] D. Bottomley, T. Ogino, Alternative to the Shuttleworth formulation of solid surface stress, Physical Review B 63 (16) (2001) 20-24.

[83] H. L. Duan, J. Wang, Z. P. Huang, B. L. Karihaloo, Size-dependent effective elastic constants of solids containing nanoinhomogeneities with interface stress, Journal of the Mechanics and Physics of Solids 53 (7) (2005) 1574-1596.

[84] Z. P. Huang, J. Wang, A theory of hyperelasticity of multi-phase media with surface/interface energy effect, Acta Mechanica $182(3-4)(2006)$ 195-210.

[85] S. G. Mogilevskaya, S. L. Crouch, H. K. Stolarski, Multiple interacting circular nano-inhomogeneities with surface/interface effects, Journal of the Mechanics and Physics of Solids 56 (6) (2008) 2298-2327.

[86] V. A. Marichev, Surface tension of solids. Structure-mechanical approach, Protection of Metals 44 (2) (2008) $105-119$.

[87] V. A. Marichev, General thermodynamic equations for the surface tension of liquids and solids, Surface Science 604 (3-4) (2010) 458-463.

[88] Z. Q. Wang, Y. P. Zhao, Z. P. Huang, The effects of surface tension on the elastic properties of nano structures, International Journal of Engineering Science 48 (2) (2010) 140-150.

[89] Z. Huang, Shape-dependent natural boundary condition of Lagrangian field, Applied Mathematics Letters 61 (2016) 56-61. 
[90] M. E. Gurtin, A. I. Murdoch, Addenda to our paper A continuum theory of elastic material surfaces, Archive for Rational Mechanics and Analysis 59 (4) (1975) 389-390.

[91] R. M. Bowen, C. C. Wang, Introduction to Vectors and Tensors: Linear and Multilinear Algebra, 1976.

[92] E. Kreyszig, Differential Geometry, Dover, 1991. 